\title{
Endpoint estimates for commutators of mutilinear square function satisfying some integrable condition
}

\author{
Dongxiang Chen*, Anzhi Huang \\ Department of Mathematic and information Science, Jiangxi Normal University, Nanchang, China. \\ Communicated by Y. Hu
}

\begin{abstract}
In this paper, the $\left(\mathrm{L}^{\mathrm{p}_{1}} \times \cdots \times \mathrm{L}^{\mathrm{p}_{\mathrm{m}}}, \mathrm{L}^{\mathrm{q}}\right)$-estimate for the commutator $\mathrm{T}_{\Pi b}$ generalized by multilinear square function $\mathrm{T}$ and Lipschitz function $\vec{b}$ is established for $\frac{1}{q}=\sum_{j=1}^{m} \frac{1}{p_{i}}-\frac{\beta}{n}, p_{i}>p_{0} \geqslant 1$. Meanwhile, we also establish $\left(L^{p_{1}} \times \cdots \times L^{p_{m}}, \dot{\Lambda}_{\beta}-\frac{n}{p}\right)-$ boundedness and $\left(L^{\frac{n}{\beta_{1}}} \times \cdots \times L^{\frac{n}{\beta m}}, B M O\right)$-estimates for the commutator $T_{\Pi b}$. Finally, the $\left(L^{p_{1}} \times \cdots \times L^{p_{m}}, \dot{\mathrm{F}}_{\mathrm{p}}^{\beta, \infty}\right)$-boundedness is obtained, too. (C) 2017 All rights reserved.
\end{abstract}

Keywords: Multilinear square function, iterated commutator, Lipschitz space, Triebel-Lizorkin space. 2010 MSC: 42B25, 42B30, 42B35.

\section{Introduction}

It is well-known that multilinear Calderon-Zygmund operators originates from the works of Coifman and Meyer's, see [1-3]. Later, Grafakos and Torres [9, 10] extended Coifman and Meyer's works. Since multilinear Littlewood-Paley g-function and related multilinear Littlewood-Paley type estimates were applied to PDE and any other fields, see [4-6], more and more people devote themselves to study the multilinear Littlewood-Paley theory and more works about multilinear Littlewood-Paley type operators, see $[13,15,17,18]$ and the references therein.

Let $K$ be a locally integrable function defined away from the diagonal $x=y_{1}=\cdots=y_{m}$ in $\left(R^{n}\right)^{m+1}$ and $\mathrm{K}_{\mathrm{t}}=\mathrm{t}^{-\mathrm{mn}} \mathrm{K}(\cdot / \mathrm{t})$. Then, multilinear square function $\mathrm{T}$ is defined by

$$
T(\vec{f})(x)=\left(\int_{0}^{\infty}\left|\int_{R^{n m}} K_{t}\left(x, y_{1}, \cdots, y_{m}\right) f_{1}\left(y_{1}\right) \cdots f_{m}\left(y_{m}\right) d \vec{y}\right|^{2} \frac{d t}{t}\right)^{\frac{1}{2}}
$$

where $\vec{f}=\left(f_{1}, \cdots, f_{m}\right) \in\left(S\left(R^{n}\right)\right)^{m}$ and $x \notin \cap_{j=1}^{m}$ supp $f_{j}$.

To study the boundedness of multilinear square function and the associated commutators, $\mathrm{Si}$, Xue and Yabuta introduced the following condition.

\footnotetext{
*Corresponding author

Email addresses: chendx020@aliyun.com (Dongxiang Chen), 18779100971@163.com (Anzhi Huang)
} 
Definition 1.1. Let $1 \leqslant p_{0}<\infty, S_{j}(Q)=2^{j} Q \backslash 2^{j-1} Q$, if $j \geqslant 1$, and $S_{0}(Q)=Q$. Then, assume that

$\left(\mathrm{H}_{1}\right)$ for all $\mathrm{p}_{0} \leqslant \mathrm{r}_{1}, \cdots, \mathrm{r}_{\mathrm{m}}<\infty$ and $0<\mathrm{r}<\infty$ with $\frac{1}{\mathrm{r}}=\frac{1}{r_{1}}+\cdots+\frac{1}{r_{\mathrm{m}}}$. T maps $\mathrm{L}^{\mathrm{r}_{1}} \times \cdots \times \mathrm{L}^{\mathrm{r}_{\mathrm{m}}}$ into $\mathrm{L}^{\mathrm{r}, \infty}$;

$\left(\mathrm{H}_{2}\right)$ there exists $\delta>\frac{n}{p_{0}}$ so that for the conjugate exponent $\mathrm{p}_{0}^{\prime}$ of $\mathrm{p}_{0}$, one has

$$
\left(\int_{S_{j_{m}}(Q)} \cdots \int_{S_{j_{1}}(Q)}\left(\int_{0}^{\infty}\left|K_{t}(x, \vec{y})-K_{t}(z, \vec{y})\right|^{2} \frac{d t}{t}\right)^{\frac{p_{0}^{\prime}}{2}} d \vec{y}\right)^{\frac{1}{p_{0}^{\prime}}} \leqslant C \frac{|x-z|^{m\left(\delta-\frac{n}{p_{0}}\right)}}{|Q|^{\frac{m}{n}}} 2^{-m \delta j_{0}}
$$

for all balls $Q$, all $x, z \in \frac{1}{2} Q$ and $\left(j_{1}, \cdots, j_{m}\right) \neq(0, \cdots, 0)$, where $j_{0}=\max _{k=1, \cdots, m}\left\{j_{k}\right\}$;

$\left(\mathrm{H}_{3}\right)$ there exists some positive constant $\mathrm{C}>0$ such that

$$
\left(\int_{S_{j_{m}}(Q)} \cdots \int_{S_{j_{1}}(Q)}\left(\int_{0}^{\infty}\left|K_{t}(x, \vec{y})\right|^{2} \frac{d t}{t}\right)^{\frac{p_{0}^{\prime}}{2}} d \vec{y}\right)^{\frac{1}{p_{0}^{\prime}}} \leqslant C \frac{2^{-m n j_{0} / p_{0}}}{|Q|^{\frac{m}{p_{0}}}}
$$

for all balls $Q$, all $x, z \in \frac{1}{2} Q$ and $\left(j_{1}, \cdots, j_{m}\right) \neq(0, \cdots, 0)$, where $j_{0}=\max _{k=1, \cdots, m}\left\{j_{k}\right\}$.

The iterated commutator of multilinear square operator T with BMO functions $b$ is defined as following:

$$
\mathrm{T}_{\Pi \mathrm{b}}(\vec{f})(x)=\left(\int_{0}^{\infty}\left|\int_{R^{n m}} \prod_{j=1}^{m}\left[b_{j}(x)-b_{j}\left(y_{j}\right)\right] K_{t}(x, \vec{y}) f_{1}\left(y_{1}\right) \cdots f_{m}\left(y_{m}\right) d \vec{y}\right|^{2} \frac{d t}{t}\right)^{\frac{1}{2}}
$$

for any $\vec{f}=\left(f_{1}, \cdots, f_{m}\right) \in S\left(R^{n}\right) \times \cdots \times S\left(R^{n}\right), \vec{y}=\left(y_{1}, \cdots, y_{m}\right)$ and $x \notin \cap_{j}$ supp $f_{j}$.

Shi et al. [15] established multiple weighted estimates for multilinear square operator $T$ with $v_{\vec{\omega}} \in$ $A_{\overrightarrow{\mathrm{P}} / \mathrm{p}_{0}}$. But we only use the following un-weighted case.

Proposition 1.2. Let $\mathrm{T}$ be the multilinear square function with a kernel satisfying conditions $\left(\mathrm{H}_{1}\right),\left(\mathrm{H}_{2}\right)$ and $(\mathrm{H} 3)$ for some $1 \leqslant p_{0}<\infty$. Then, for any $\mathrm{p}_{0} \leqslant \mathrm{p}_{1}, \cdots, \mathrm{p}_{\mathrm{m}}<\infty$, satisfying $\frac{1}{\mathrm{p}}=\frac{1}{\mathrm{p}_{1}}+\cdots+\frac{1}{\mathrm{p}_{\mathrm{m}}}$, the following estimates hold.

(i) If there is no $p_{i}=p_{0}$, then

$$
\|T(\vec{f})\|_{L^{p}\left(R^{n}\right)} \leqslant C \prod_{j=1}^{m}\left\|f_{j}\right\|_{L^{p_{j}\left(R^{n}\right)}}
$$

(ii) If there is some $p_{i}=p_{0}$, then

$$
\|T(\vec{f})\|_{L^{p, \infty}\left(R^{n}\right)} \leqslant C \prod_{j=1}^{m}\left\|f_{j}\right\|_{L^{p_{j}}\left(R^{n}\right)}
$$

In order to illustrate our motivation, we define the multilinear fractional integral

$$
I_{m, \alpha} f(x)=\int_{\left(R^{n}\right)^{m}} \frac{f_{1}\left(y_{1}\right) \cdots f_{m}\left(y_{m}\right)}{\left(\left|x-y_{1}\right|+\cdots+\left|x-y_{m}\right|\right)^{m n-\alpha}} d y_{1} \cdots d y_{m}
$$

where $m \geqslant 2$ and $0<\alpha<m n$.

It is well-known that multilinear fractional integral was first studied by Grafakos [8]. Later Kenig and Stein [11] showed that multilinear fractional operator $I_{m, \alpha}$ is bounded from $L^{p_{1}} \times \cdots \times L^{p_{m}}$ to $L^{q}\left(R^{n}\right)$ 
with $\frac{1}{q}=\sum_{i=1}^{m} \frac{1}{p_{i}}-\frac{\alpha}{n}$ and each $p_{i}>1$. If some $p_{i}=1$, then $I_{m, \alpha}$ is bounded from $L^{p_{1}} \times \cdots \times L^{p_{m}}$ to $L^{q, \infty}\left(R^{\mathfrak{n}}\right)$. In 2008, Tang [16] established the $\left(M_{\mathbf{p}_{1}}^{q_{1}}\left(R^{\mathfrak{n}}\right) \times \cdots \times M_{\mathcal{p}_{m}}^{q_{m}}\left(R^{\mathfrak{n}}\right), B M O\right)$ estimate and $\left(M_{\mathbf{p}_{1}}^{q_{1}}\left(R^{\mathfrak{n}}\right) \times\right.$ $\left.\cdots \times M_{p_{m}}^{q_{m}}\left(R^{n}\right), \operatorname{Lip}_{\beta-\frac{n}{q}}\right)$-estimates for multilinear fractional integral operator $I_{m, \alpha}$.

The purpose of this paper is to investigate some endpoint estimates for iterated commutators generalized by multilinear square function $T$ and Lipschitz function $b$. Since the kernel of multilinear square operator does not have the explicit pointwise size condition, we cannot obtain our results via the same boundedness of multilinear fractional integrals after using Minkowski's inequality. We are not able to use the original approach (for instance, one often proves that the commutator is dominated by multilinear fractional integrals) so that we need to seek new methods. For this reason, our computations and estimates in proofs are complicated and possibly very technical. We formulate our results as follows.

Theorem 1.3. Let $\mathrm{T}$ be a multilinear square function with a kernel satisfying conditions $\left(\mathrm{H}_{1}\right),\left(\mathrm{H}_{2}\right)$ and $\left(\mathrm{H}_{3}\right)$ for some $1 \leqslant p_{0}<\infty$. Assume that $\beta=\sum_{j=1}^{m} \beta_{j}$ with $0<\beta_{j}<1$ and $1<q_{j}, p_{i}<\infty$ satisfying

$$
\frac{1}{q_{j}}=\sum_{j=1}^{m} \frac{1}{p_{j}}-\frac{\beta_{j}}{n}, \quad \frac{1}{q}=\sum_{j=1}^{m} \frac{1}{q_{j}}, \quad \frac{1}{p}=\sum_{j=1}^{m} \frac{1}{p_{j}} .
$$

Then for $b_{i} \in \dot{\Lambda}_{\beta_{i}}$, we have if all $p_{i}>p_{0}$, then

$$
\left\|T_{\Pi b}(\vec{f})\right\|_{L q\left(R^{n}\right)} \leqslant C \prod_{j=1}^{m}\left\|b_{j}\right\|_{\dot{\lambda}_{\beta_{j}}}\left\|f_{j}\right\|_{L^{p_{j}\left(R^{n}\right)}} .
$$

The following theorem is concerned with the mapping properties in Lipschitz spaces.

Theorem 1.4. Let $\mathrm{T}$ be a multilinear square function with a kernel satisfying conditions $\left(\mathrm{H}_{1}\right),\left(\mathrm{H}_{2}\right)$ and $\left(\mathrm{H}_{3}\right)$ for some $1 \leqslant p_{0}<\infty$. Assume that $\mathrm{p}_{0}<\mathrm{p}_{1}, \cdots, \mathrm{p}_{\mathrm{m}}$ satisfying $\frac{1}{\mathrm{p}}=\frac{1}{\mathrm{p}_{1}}+\cdots+\frac{1}{\mathrm{p}_{\mathrm{m}}}, \mathrm{p}_{\mathrm{i}}>\frac{\mathrm{n}}{\beta_{\mathrm{i}}}$ and $\delta>\max \left\{\frac{\mathfrak{n}}{p_{0}}, \frac{\beta}{2}-n\left(\frac{1}{p_{0}}-\frac{1}{2 p}\right)\right\}$. Then for $b_{i} \in \dot{\Lambda}_{\beta_{i}}$ for $i=1, \cdots, m$ with $\beta=\sum_{i=1}^{m} \beta_{i}$, we have

$$
\left\|T_{\Pi b}(\vec{f})\right\|_{\dot{\Lambda}_{\beta-\frac{n}{p}}} \leqslant C \prod_{j=1}^{m}\left\|b_{j}\right\|_{\dot{\Lambda}_{\beta_{j}}}\left\|f_{j}\right\|_{L^{p_{j}}} .
$$

In order to obtain the following results, we introduce some new condition:

Condition $\left(\mathrm{H}_{4}\right)$ : There exists some positive constant $\mathrm{C}>0$ such that

$$
\left(\int_{S_{0}(Q)} \cdots \int_{S_{0}(Q)}\left(\int_{0}^{\infty}\left|K_{t}(x, \vec{y})\right|^{2} \frac{d t}{t}\right)^{\frac{p_{0}^{\prime}}{2}} d \vec{y}\right)^{\frac{1}{p_{0}^{\prime}}} \leqslant C|Q|^{-\frac{m}{p_{0}}}
$$

for all balls $Q$.

Theorem 1.5 says the $\left(\mathrm{L}^{\frac{n}{\beta_{1}}} \times \cdots \times \mathrm{L}^{\frac{\mathrm{n}}{\beta_{m}}}, \mathrm{BMO}\right)$-boundedness of the iterated commutator $\mathrm{T}_{\Pi \mathrm{b}}$.

Theorem 1.5. Let $\mathrm{T}$ be a multilinear square function with a kernel satisfying conditions $\left(\mathrm{H}_{1}\right),\left(\mathrm{H}_{2}\right),\left(\mathrm{H}_{3}\right)$ and $\left(\mathrm{H}_{4}\right)$ for some $1 \leqslant p_{0}<\min \left\{\frac{n}{\beta_{1}}, \cdots, \frac{n}{\beta_{m}}\right\}$. We further assume that $\delta>\frac{n}{p_{0}}$. Then for $b_{i} \in \dot{\Lambda}_{\beta_{i}}$ for $i=1, \cdots, m$ with $\beta=\sum_{i=1}^{m} \beta_{i}$, we have

$$
\left\|T_{\Pi b}(\vec{f})\right\|_{B M O} \leqslant C \prod_{j=1}^{m}\left\|b_{j}\right\|_{\dot{\Lambda}_{\beta_{j}}}\left\|f_{j}\right\|_{L^{\frac{n}{\beta_{j}}}} .
$$

Finally we establish the $\left(\mathrm{L}^{\mathrm{p}_{1}} \times \cdots \times \mathrm{L}^{\mathrm{p}_{\mathrm{m}}}, \dot{\mathrm{F}}_{\mathrm{p}}^{\beta, \infty}\right)$-boundedness of $\mathrm{T}_{\Pi \mathrm{b}}$ with $\frac{1}{\mathrm{p}_{1}}+\cdots+\frac{1}{\mathrm{p}_{\mathrm{m}}}=\frac{1}{\mathrm{p}}$. 
Theorem 1.6. Let $\mathrm{T}$ be a multilinear square function with a kernel satisfying conditions $\left(\mathrm{H}_{1}\right),\left(\mathrm{H}_{2}\right),\left(\mathrm{H}_{3}\right)$ and $\left(\mathrm{H}_{4}\right)$ for some $1 \leqslant p_{0}<\min \left\{\frac{n}{\beta_{1}}, \cdots, \frac{n}{\beta_{m}}\right\}$. We further assume that $\delta>\max \left\{\frac{n}{p_{0}}, \frac{\beta}{2}-\frac{n}{p_{0}}\right\}$. Then for $b_{i} \in \dot{\Lambda}_{\beta_{i}}$ for $i=1, \cdots$, with $\beta=\sum_{i=1}^{m} \beta_{i}$, we have

$$
\left\|T_{\Pi b}(\vec{f})\right\|_{\dot{F}_{p}^{\beta, \infty}} \leqslant C \prod_{j=1}^{m}\left\|b_{j}\right\|_{i_{\beta_{j}}}\left\|f_{j}\right\|_{L^{p_{j}}}
$$

Remark 1.7. In our knowledge, we first investigate the endpoint estimates for the commutator generalized by multilinear square function and Lipschitz function. By the assumption on the kernel of the multilinear square function, the commutator that we study cannot be dominated by multilinear fractional integral. So our results cannot be followed from the results in [16]. The computation and proof of our results are more complicated with more techniques. On the other hand, the boundedness of commutator in Triebel-Lizorkin space is also new and interesting.

This paper is arranged as follows. In Section 2, we will give some basic notations and use lemmas. The $\left(\mathrm{L}^{\mathrm{p}_{1}} \times \cdots \times \mathrm{L}^{\mathrm{p}_{\mathrm{m}}}, \mathrm{L}^{\mathrm{q}}\right)$-boundedness of the commutator will be given in Section 3 . We will investigate the Lipschitz mapping property in Section 4 and the $\left(\mathrm{L}^{\frac{n}{\beta_{1}}} \times \cdots \times \mathrm{L}^{\frac{n}{\beta_{m}}}, \mathrm{BMO}\right)$-boundedness of $\mathrm{T}_{\Pi \mathrm{b}}$ will be proved in Section 5. Finally, we will establish the $\left(\mathrm{L}^{\mathrm{p}_{1}} \times \cdots \times \mathrm{L}^{\mathrm{p}_{\mathrm{m}}}, \dot{\mathrm{F}}_{\mathrm{p}}^{\beta, \infty}\right)$-boundedness of the iterated commutator of multilinear square function.

\section{Preliminary and some lemmas}

For $\beta>0$, the homogeneous Lipschitz space $\dot{\Lambda}_{\beta}\left(R^{n}\right)$ is the space of function $f$ such that

$$
\|\mathrm{b}\|_{\dot{\Lambda}_{\beta}}=\sup _{x, h \in R^{n}, h \neq 0} \frac{\left|\Delta_{h}^{[\beta]+1}\right| \mathrm{b}(x)}{|h|^{\beta}}<\infty,
$$

where $\Delta_{h}^{k}$ is the k-th difference operator, which is referred in [14].

Here are some information on this Lipschitz spaces. They can be found in [14].

\section{Lemma 2.1.}

(a) For $0<\beta<1,1 \leqslant q \leqslant \infty$, we have

$$
\|\mathrm{f}\|_{\dot{\Lambda}_{\beta}} \approx \sup _{\mathrm{Q}} \frac{1}{|\mathrm{Q}|^{1+\frac{\beta}{n}}} \int_{\mathrm{Q}}\left|\mathrm{f}(\mathrm{x})-\mathrm{f}_{\mathrm{Q}}\right| \mathrm{d} x \approx \sup _{\mathrm{Q}} \frac{1}{|\mathrm{Q}|^{\frac{\beta}{n}}}\left(\frac{1}{|\mathrm{Q}|} \int_{\mathrm{Q}}\left|\mathrm{f}(\mathrm{x})-\mathrm{f}_{\mathrm{Q}}\right|^{\mathrm{q}} \mathrm{d} x\right)^{\frac{1}{q}} .
$$

For $\mathrm{q}=\infty$, the formula should be interpreted appropriately.

(b) Let $\mathrm{Q} \subset \mathrm{Q}^{*}$, then

$$
\left|f_{Q}-f_{Q^{*}}\right| \leqslant C\|f\|_{\dot{\Lambda}_{\beta}}|Q|^{\frac{\beta}{n}}
$$

where $\mathrm{f}_{\mathrm{Q}}=\frac{1}{|\mathrm{Q}|} \int_{\mathrm{Q}} \mathrm{f}(\mathrm{x}) \mathrm{d} \mathrm{x}$.

In fact, if $x \in \mathrm{Q} \subset \mathrm{Q}^{*}$, it is easy to follow that

$$
\left|b(x)-b_{Q^{*}}\right| \leqslant C\|f\|_{\dot{\Lambda}_{\beta}}\left|Q^{*}\right|^{\frac{\beta}{n}},
$$

which will be used frequently in our the proof of our theorems.

Next we will give an equivalent norm of the Triebel-Lizorkin space $\dot{F}_{p}^{\beta, \infty}$. 


\section{Lemma 2.2.}

$$
\|\mathbf{f}\|_{\dot{\mathrm{F}}_{\mathrm{p}}^{\beta, \infty}} \approx\left\|\sup _{\mathrm{Q} \ni \cdot|\mathrm{Q}|^{1+\frac{\beta}{n}}} \int_{\mathrm{Q}}\left|\mathrm{f}(x)-\mathrm{f}_{\mathrm{Q}}\right| \mathrm{d} x\right\|_{\mathrm{L}^{\mathrm{p}}}
$$

Let

$$
M_{\delta} f(x)=M\left(|f|^{\delta}\right)^{\frac{1}{\delta}}(x)=\sup _{Q \ni x}\left(\frac{1}{|Q|} \int_{Q}|f(y)|^{\delta} d y\right)^{\frac{1}{\delta}}
$$

Also, let $M^{\sharp}$ be the usual sharp maximal function of Fefferman and Stein [7],

$$
M^{\sharp} f(x)=\sup _{Q \ni x} \inf _{c} \frac{1}{|Q|} \int_{Q}|f(y)-c| d y \approx \sup _{Q \ni x} \frac{1}{|Q|} \int_{Q}\left|f(y)-f_{Q}\right| d y,
$$

where $f_{Q}=\frac{1}{|Q|} \int_{Q} f(y) d y$.

We need to use the following Fefferman-Stein inequality, see [7].

Lemma 2.3. Let $0<p, \delta<\infty$ and let $\omega$ be a weight in $A_{\infty}$. Then, there exists $C>0$ (depending on the $A_{\infty}$ constant of $\omega)$, such that

$$
\int_{R^{n}}\left(M_{\delta} f(x)\right)^{p} \omega(x) d x \leqslant C \int_{R^{n}}\left(M_{\delta}^{\sharp} f(x)\right)^{p} \omega(x) d x
$$

for all functions $\mathrm{f}$ for which the left hand side is finite.

We will use unweighted case in the proof of Theorem 1.3.

Now we give the definition of fractional maximal operators as follows:

$$
M_{\alpha} f(x)=\sup _{Q \ni x} \frac{1}{|Q|^{1-\frac{\alpha}{n}}} \int_{Q}|f(y)| d y,
$$

and

$$
M_{\alpha, s} f(x)=\sup _{Q \ni x}\left(\frac{1}{|Q|^{1-\frac{\alpha s}{n}}} \int_{Q}|f(y)|^{s} d y\right)^{\frac{1}{s}}
$$

From [8], we know that:

Lemma 2.4. Let $0<\beta<n, 0<s<p<\frac{n}{\alpha}, \frac{1}{q}=\frac{1}{p}-\frac{\alpha}{n}$. Then

$$
\left\|M_{\alpha, s}(f)\right\|_{L^{q}\left(R^{n}\right)} \leqslant C\|f\|_{L^{p}\left(R^{n}\right)} .
$$

Definition 2.5. For $f$ a complex-valued locally integrable function on $R^{n}$, set

$$
\|f\|_{B M O}=\sup _{Q} \frac{1}{|Q|} \int_{Q}\left|f(x)-f_{Q}\right| d x
$$

where the supremum is taken over all cubes $Q$ in $R^{n}$.

\section{The boundedness of iterated commutator of multilinear square functions on Lebesgue spaces}

In this section, we will investigate the $\left(\mathrm{L}^{\mathrm{p}_{1}} \times \cdots \times \mathrm{L}^{\mathrm{p}_{\mathrm{m}}}, \mathrm{L}^{\mathrm{q}}\right)$-boundedness for commutators of square function.

We first establish the following pointwise estimate of sharp maximal function of multilinear square function. 
Lemma 3.1. Let $\mathrm{T}_{\Pi \mathrm{b}}$ be the iterated commutator defined as (1.1) with kernel $\mathrm{K}_{\mathrm{t}}$ satisfying the conditions $\left(\mathrm{H}_{1}\right),\left(\mathrm{H}_{2}\right)$ and $\left(\mathrm{H}_{3}\right)$. Assume that $0<\delta<\min \left\{1, \frac{p_{0}}{\mathrm{~m}}\right\}$ and $\mathrm{b}_{j} \in \dot{\Lambda}_{\beta_{j}}$ with $\beta=\beta_{1}+\cdots+\beta_{\mathrm{m}}, 0<\beta_{j}<1,0 \leqslant \beta<1$. Then

$$
M_{\delta}^{\sharp}\left(T_{\Pi \mathbf{b}}(\vec{f})(x) \leqslant C \prod_{j=1}^{m}\left\|b_{j}\right\|_{\dot{\Lambda}_{\beta_{j}}}\left(M_{\beta}\left(T(\vec{f})(x)+\prod_{j=1}^{m} M_{\beta_{j}, p_{0}}\left(f_{j}\right)(x)\right) .\right.\right.
$$

Proof. For brief, we only need to consider the case $m=2$ since the argument for $m>2$ is similar. Fix a point $x \in R^{n}$ and a ball $Q$ containing $x$. For $0<\delta<\min \left\{1, \frac{p_{0}}{2}\right\}$, we split $f_{i}$ into the following form $f_{i}=f_{i} \chi Q^{*}+f_{i} \chi_{R^{n}} \backslash Q^{*}=f_{i}^{0}+f_{i}^{\infty}$, where $Q^{*}=2 Q$. It suffices to show that there exists a constant $c_{Q}$ such that

$$
\begin{aligned}
\left(\left.\frac{1}{|\mathrm{Q}|} \int_{\mathrm{Q}}|| \mathrm{T}_{\Pi \mathrm{b}}\left(\mathrm{f}_{1}, \mathrm{f}_{2}\right)(z)\right|^{\delta}-\left|\mathrm{C}_{\mathrm{Q}}\right|^{\delta} \mid \mathrm{d} z\right)^{\frac{1}{\delta}} \leqslant & C\left(\frac{1}{|\mathrm{Q}|} \int_{\mathrm{Q}}\left|\mathrm{T}_{\Pi \mathrm{b}}\left(\mathrm{f}_{1}, \mathrm{f}_{2}\right)(z)-\mathrm{C}_{\mathrm{Q}}\right|^{\delta} \mathrm{d} z\right)^{\frac{1}{\delta}} \\
\leqslant & C\left(\frac{1}{|\mathrm{Q}|} \int_{\mathrm{Q}}\left|\mathrm{b}_{1}(z)-\left(\mathrm{b}_{1}\right)_{\mathrm{Q}^{*}}\right|\left|\mathrm{b}_{2}(z)-\left(\mathrm{b}_{2}\right)_{\mathrm{Q}^{*}}\right|\left|\mathrm{T}\left(\mathrm{f}_{1}, \mathrm{f}_{2}\right)(z)\right| \mathrm{d} z\right) \\
& +\mathrm{C}\left(\frac{1}{|\mathrm{Q}|} \int_{\mathrm{Q}}\left|\mathrm{b}_{1}(z)-\left(\mathrm{b}_{1}\right)_{\mathrm{Q}^{*}}\right|^{\delta}\left|\mathrm{T}\left(\mathrm{f}_{1},\left(\mathrm{~b}_{2}-\left(\mathrm{b}_{2}\right)_{\mathrm{Q}^{*}}\right) \mathrm{f}_{2}\right)(z)\right|^{\delta} \mathrm{d} z\right)^{\frac{1}{\delta}} \\
& +\mathrm{C}\left(\frac{1}{|\mathrm{Q}|} \int_{\mathrm{Q}}\left|\mathrm{b}_{2}(z)-\left(\mathrm{b}_{2}\right)_{\mathrm{Q}^{*}}\right|^{\delta}\left|\mathrm{T}\left(\left(\mathrm{b}_{1}-\left(\mathrm{b}_{1}\right)_{\mathrm{Q}^{*}}\right) \mathrm{f}_{1}, \mathrm{f}_{2}\right)(z)\right|^{\delta} \mathrm{d} z\right)^{\frac{1}{\delta}} \\
& +\mathrm{C}\left(\frac{1}{|\mathrm{Q}|} \int_{\mathrm{Q}}\left|\mathrm{T}\left(\left(\mathrm{b}_{1}-\left(\mathrm{b}_{1}\right)_{\mathrm{Q}^{*}}\right) \mathrm{f}_{1},\left(\mathrm{~b}_{2}-\left(\mathrm{b}_{2}\right)_{\mathrm{Q}^{*}}\right) \mathrm{f}_{2}\right)(z)-\mathrm{C}_{\mathrm{Q}}\right|^{\delta} \mathrm{d} z\right)^{\frac{1}{\delta}} \\
= & \mathrm{I}+\mathrm{II}+\mathrm{III}+\mathrm{IV} .
\end{aligned}
$$

We first estimate I. Lemma 2.1 says that

$$
I \leqslant C\left\|b_{1}\right\|_{\operatorname{Lip}_{\beta_{1}}}\left\|b_{2}\right\|_{\operatorname{Lip}_{\beta_{2}}} M_{\beta}\left(T\left(f_{1}, f_{2}\right)\right)(x) .
$$

Since II and III are very symmetric, we only consider the second term II. We decompose

$$
\begin{aligned}
& \mathrm{II} \leqslant \mathrm{C}\left(\frac{1}{|\mathrm{Q}|} \int_{\mathrm{Q}}\left|\mathrm{b}_{1}(z)-\left(\mathrm{b}_{1}\right)_{\mathrm{Q}^{*}}\right|^{\delta}\left|\mathrm{T}\left(\mathrm{f}_{1}^{0},\left(\mathrm{~b}_{2}-\left(\mathrm{b}_{2}\right)_{\mathrm{Q}^{*}}\right) \mathrm{f}_{2}^{0}\right)(z)\right|^{\delta} \mathrm{d} z\right)^{\frac{1}{\delta}} \\
& +\mathrm{C}\left(\frac{1}{|\mathrm{Q}|} \int_{\mathrm{Q}}\left|\mathrm{b}_{1}(z)-\left(\mathrm{b}_{1}\right)_{\mathrm{Q}^{*}}\right|^{\delta}\left|\mathrm{T}\left(\mathrm{f}_{1}^{0},\left(\mathrm{~b}_{2}-\left(\mathrm{b}_{2}\right)_{\mathrm{Q}^{*}}\right) \mathrm{f}_{2}^{\infty}\right)(z)\right|^{\delta} \mathrm{d} z\right)^{\frac{1}{\delta}} \\
& +\mathrm{C}\left(\frac{1}{|\mathrm{Q}|} \int_{\mathrm{Q}}\left|\mathrm{b}_{1}(z)-\left(\mathrm{b}_{1}\right)_{\mathrm{Q}^{*}}\right|^{\delta}\left|\mathrm{T}\left(\mathrm{f}_{1}^{\infty},\left(\mathrm{b}_{2}-\left(\mathrm{b}_{2}\right)_{\mathrm{Q}^{*}}\right) \mathrm{f}_{2}^{0}\right)(z)\right|^{\delta} \mathrm{d} z\right)^{\frac{1}{\delta}} \\
& +C\left(\frac{1}{|Q|} \int_{\mathrm{Q}}\left|\mathrm{b}_{1}(z)-\left(\mathrm{b}_{1}\right)_{\mathrm{Q}^{*}}\right|^{\delta}\left|\mathrm{T}\left(f_{1}^{\infty},\left(\mathrm{b}_{2}-\left(\mathrm{b}_{2}\right) \mathrm{Q}^{*}\right) \mathrm{f}_{2}^{\infty}\right)(z)\right|^{\delta} \mathrm{d} z\right)^{\frac{1}{\delta}} \\
& =\mathrm{II}_{1}+\mathrm{II}_{2}+\mathrm{II}_{3}+\mathrm{II}_{4} \text {. }
\end{aligned}
$$

Proposition 1.2, (2.1) and the Kolmogorov inequality $\left(\mathrm{p}_{0}<\mathrm{q}_{0}\right)$ give that

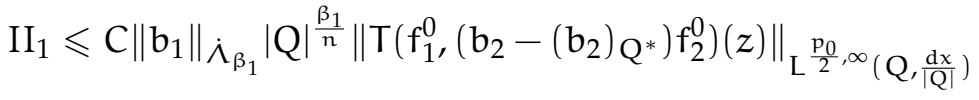

$$
\begin{aligned}
& \leqslant C\left\|b_{1}\right\|_{\lambda_{\beta_{1}}}|Q|^{\frac{\beta_{1}}{n}}\left(\frac{1}{|Q|} \int_{Q}\left|f_{1}\left(y_{1}\right)\right|^{p_{0}} d y_{1}\right)^{1 / p_{0}}\left(\frac{1}{|Q|} \int_{Q}\left|b_{2}\left(y_{2}\right)-\left(b_{2}\right)_{Q^{*}}\right|^{p_{0}}\left|f_{1}\left(y_{1}\right)\right|^{p_{0}} d y_{1}\right)^{1 / p_{0}} \\
& \leqslant C\left\|b_{1}\right\|_{\dot{\Lambda}_{\beta_{1}}}\left\|b_{2}\right\|_{{\dot{\beta_{\beta}}}_{\beta_{2}}} M_{\beta_{1}, p_{0}}\left(f_{1}\right)(x) M_{\beta_{2}, p_{0}}\left(f_{2}\right)(x) \text {. }
\end{aligned}
$$


Since $\mathrm{II}_{2}$ and $\mathrm{II}_{3}$ are symmetric, we only bound the second term $\mathrm{II}_{2}$. Thanks to Minkowski's inequality and the condition $\left(\mathrm{H}_{3}\right)$, we obtain

$$
\begin{aligned}
& \mathrm{II}_{2} \leqslant \mathrm{C}\left\|\mathrm{b}_{1}\right\|_{{\dot{\lambda_{\beta}}}_{\beta_{1}}}|\mathrm{Q}|^{\frac{\beta_{1}}{n}} \frac{1}{|\mathrm{Q}|} \int_{\mathrm{Q}} \int_{\mathrm{R}^{2 n}}\left(\int_{0}^{\infty}\left|\mathrm{K}_{\mathrm{t}}\left(z, \mathrm{y}_{1}, \mathrm{y}_{2}\right)\right|^{2} \frac{\mathrm{dt}}{\mathrm{t}}\right)^{\frac{1}{2}}\left|\mathrm{f}_{1}^{\infty}\left(\mathrm{y}_{1}\right)\left\|\left(\mathrm{b}_{2}\left(\mathrm{y}_{2}\right)-\left(\mathrm{b}_{2}\right)\right)_{\mathrm{Q}^{*}}\right\| \mathrm{f}_{2}^{0}\left(\mathrm{y}_{2}\right)\right| \mathrm{d} \mathrm{y}_{1} \mathrm{~d} \mathrm{y}_{2} \mathrm{~d} z \\
& \left.\leqslant C\left\|b_{1}\right\|_{{\dot{\beta_{\beta}}}_{1}}|\mathrm{Q}|^{\frac{\beta_{1}}{n}} \frac{1}{|\mathrm{Q}|} \int_{\mathrm{Q}_{\mathrm{k}=1}} \sum_{\mathrm{S}_{0}\left(\mathrm{Q}^{*}\right) \times \mathrm{S}_{\mathrm{k}}\left(\mathrm{Q}^{*}\right)}\left(\int_{0}^{\infty}\left|\mathrm{K}_{\mathrm{t}}\left(z, \mathrm{y}_{1}, \mathrm{y}_{2}\right)\right|^{2} \frac{\mathrm{dt}}{\mathrm{t}}\right)^{\frac{\mathrm{p}_{0}^{\prime}}{2}} \mathrm{~d} \mathrm{y}_{1} \mathrm{~d} \mathrm{y}_{2}\right)^{1 / \mathrm{p}_{0}^{\prime}} \mathrm{d} z \\
& \times\left(\int_{2^{k} Q^{*} \backslash 2^{k-1} Q^{*}}\left|f_{1}\left(y_{1}\right)\right|^{p_{0}} d y_{1}\right)^{1 / p_{0}}\left(\left.\int_{Q^{*}}\left|\left(b_{2}\left(y_{2}\right)-\left(b_{2}\right)\right)_{Q^{*}} p^{p_{0}}\right| f_{2}\left(y_{2}\right)\right|^{p_{0}} d y_{2}\right)^{1 / p_{0}} \\
& \leqslant C\left\|b_{1}\right\|_{\lambda_{\beta_{1}}}\left\|b_{2}\right\|_{\lambda_{\beta_{2}}} \sum_{k=1}^{\infty} \frac{2^{-2 n k / p_{0}}}{\left|Q^{*}\right|^{\frac{2}{p_{0}}}}|Q|^{\frac{\beta_{1}}{n}}\left(\int_{2^{k} Q^{*}}\left|f_{1}\left(y_{1}\right)\right|^{p_{0}} d y_{1}\right)^{1 / p_{0}}\left|Q^{*}\right|^{\frac{\beta_{2}}{n}}\left(\int_{Q^{*}}\left|f_{2}\left(y_{2}\right)\right|^{p_{0}} d y_{2}\right)^{1 / p_{0}} \\
& \leqslant C\left\|b_{1}\right\|_{{\dot{\beta_{\beta}}}_{\beta_{1}}}\left\|b_{2}\right\|_{{\dot{\lambda_{\beta_{2}}}}_{1}} M_{\beta_{1}, p_{0}}\left(f_{1}\right)(x) M_{\beta_{2}, p_{0}}\left(f_{2}\right)(x) \text {. }
\end{aligned}
$$

Similarly, we have

$$
\begin{aligned}
& \mathrm{II}_{3} \leqslant \mathrm{C}\left\|\mathrm{b}_{1}\right\|_{{\dot{\Lambda}}_{\beta_{1}}}\left\|\mathrm{~b}_{2}\right\|_{\dot{\Lambda}_{\beta_{2}}} \sum_{\mathrm{k}=1}^{\infty} \frac{2^{-2 n k / p_{0}}}{\left|\mathrm{Q}^{*}\right|^{\frac{2}{p_{0}}}}|\mathrm{Q}|^{\frac{\beta_{1}}{n}}\left(\int_{\mathrm{Q}^{*}}\left|f_{1}\left(y_{1}\right)\right|^{p_{0}} \mathrm{~d} y_{1}\right)^{1 / \mathrm{p}_{0}}\left|\mathrm{Q}^{*}\right|^{\frac{\beta_{2}}{n}}\left(\int_{2^{k} \mathrm{Q}^{*}}\left|\mathrm{f}_{2}\left(\mathrm{y}_{2}\right)\right|^{\mathrm{p}_{0}} \mathrm{~d} \mathrm{y}_{2}\right)^{1 / \mathrm{p}_{0}} \\
& \leqslant C\left\|b_{1}\right\|_{i_{\beta_{1}}}\left\|b_{2}\right\|_{\lambda_{\beta_{2}}} M_{\beta_{1}, p_{0}}\left(f_{1}\right)(x) M_{\beta_{2}, p_{0}}\left(f_{2}\right)(x) .
\end{aligned}
$$

Next we will handle the last term $\mathrm{II}_{4}$. The condition $\left(\mathrm{H}_{1}\right)$ and Hölder's inequality give that

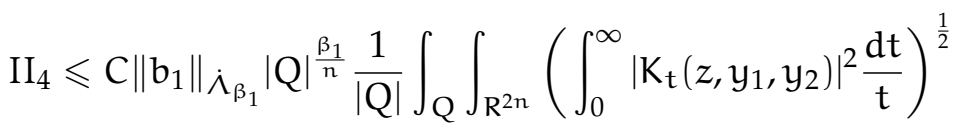

$$
\begin{aligned}
& \times\left|f_{1}^{\infty}\left(y_{1}\right)\left\|\left(b_{2}\left(y_{2}\right)-\left(b_{2}\right)\right)_{Q^{*}}\right\| f_{2}^{\infty}\left(y_{2}\right)\right| d y_{1} d y_{2} d z \\
& \leqslant C\left\|b_{1}\right\|_{\lambda_{\beta_{1}}}|Q|^{\frac{\beta_{1}}{n}} \frac{1}{|Q|} \int_{Q_{k=1}}^{\infty}\left(\int_{S_{k}\left(Q^{*}\right) \times S_{k}\left(Q^{*}\right)}\left(\int_{0}^{\infty}\left|K_{t}\left(z, y_{1}, y_{2}\right)\right|^{2} \frac{d t}{t}\right)^{\frac{p_{0}^{\prime}}{2}} d y_{1} d y_{2}\right)^{1 / p_{0}^{\prime}} d z \\
& \times\left(\int_{2^{k} Q^{*}}\left|f_{1}\left(y_{1}\right)\right|^{p_{0}} d y_{1}\right)^{1 / p_{0}}\left(\int_{2^{k} Q^{*}}\left|\left(b_{2}\left(y_{2}\right)-\left(b_{2}\right)\right)_{Q^{*}}\right| p_{0}\left|f_{2}\left(y_{2}\right)\right|^{p_{0}} d y_{2}\right)^{1 / p_{0}} \\
& \leqslant\left. C\left\|b_{1}\right\|_{\lambda_{\beta_{1}}}\left\|b_{2}\right\|_{\lambda_{\beta_{2}}} \sum_{k=1}^{\infty} \frac{2^{-2 n k / p_{0}}}{\left|Q^{*}\right|^{\frac{2}{p_{0}}}}\left|Q^{\mid \frac{\beta_{1}}{n}}\left(\int_{2^{k} Q^{*}}\left|f_{1}\left(y_{1}\right)\right|^{p_{0}} d y_{1}\right)^{1 / p_{0}}\right| Q^{*}\right|^{\frac{\beta_{2}}{n}}\left(\int_{Q^{*}}\left|f_{2}\left(y_{2}\right)\right|^{p_{0}} d y_{2}\right)^{1 / p_{0}} \\
& \leqslant C\left\|b_{1}\right\|_{i_{\beta_{1}}}\left\|b_{2}\right\|_{\lambda_{\beta_{2}}} M_{\beta_{1}, p_{0}}\left(f_{1}\right)(x) M_{\beta_{2}, p_{0}}\left(f_{2}\right)(x),
\end{aligned}
$$

which together with the estimates of $\mathrm{II}_{1}, \mathrm{II}_{2}, \mathrm{II}_{3}$ and $\mathrm{II}_{4}$ concludes that

$$
\mathrm{II} \leqslant \mathrm{C}\left\|\mathrm{b}_{1}\right\|_{{\dot{\lambda_{\beta}}}_{\beta_{1}}}\left\|\mathrm{~b}_{2}\right\|_{{\dot{\lambda_{\beta_{2}}}}_{2}} M_{\beta_{1}, p_{0}}\left(f_{1}\right)(x) M_{\beta_{2}, p_{0}}\left(f_{2}\right)(x) .
$$

Finally, we will bound the last term IV. Choosing

$$
C_{Q}=\left(\int_{0}^{\infty}\left|\int_{R^{2 n}} K_{t}\left(x_{Q}, y_{1}, y_{2}\right) \prod_{j=1}^{2}\left(b_{j}\left(y_{j}\right)-\left(b_{j}\right)_{Q^{*}}\right) f_{j}^{\infty}\left(y_{j}\right) d y_{1} d y_{2}\right|^{2} \frac{d t}{t}\right)^{\frac{1}{2}},
$$

we decompose IV into the following form:

$$
I V \leqslant C\left(\frac{1}{|Q|} \int_{Q}\left|T\left(\left(b_{1}-\left(b_{1}\right) Q^{*}\right) f_{1}^{0},\left(b_{2}-\left(b_{2}\right)_{Q^{*}}\right) f_{2}^{0}\right)(z)\right|^{\delta} d z\right)^{\frac{1}{\delta}}
$$




$$
\begin{aligned}
& +C\left(\frac{1}{|Q|} \int_{Q}\left|T\left(\left(b_{1}-\left(b_{1}\right)_{Q^{*}}\right) f_{1}^{0},\left(b_{2}-\left(b_{2}\right)_{Q^{*}}\right) f_{2}^{\infty}\right)(z)\right|^{\delta} d z\right)^{\frac{1}{\delta}} \\
& +C\left(\frac{1}{|Q|} \int_{Q}\left|T\left(\left(b_{1}-\left(b_{1}\right)_{Q^{*}}\right) f_{1}^{\infty},\left(b_{2}-\left(b_{2}\right)_{Q^{*}}\right) f_{2}^{0}\right)(z)\right|^{\delta} d z\right)^{\frac{1}{\delta}} \\
& +C\left(\frac{1}{|Q|} \int_{Q}\left|T\left(\left(b_{1}-\left(b_{1}\right)_{Q^{*}}\right) f_{1}^{\infty},\left(b_{2}-\left(b_{2}\right)_{Q^{*}}\right) f_{2}^{\infty}\right)(z)-C_{Q}\right|^{\delta} d z\right)^{\frac{1}{\delta}} \\
& =I V_{1}+I V_{2}+I V_{3}+I V_{4} .
\end{aligned}
$$

To bound $\mathrm{IV}_{1}$ : Following the same idea to estimate $\mathrm{II}_{1}$, we show that

$$
I V_{1} \leqslant C\left\|b_{1}\right\|_{i_{\beta_{1}}}\left\|b_{2}\right\|_{{\dot{\beta_{\beta}}}_{\beta_{2}}} M_{\beta_{1}, p_{0}}\left(f_{1}\right)(x) M_{\beta_{2}, p_{0}}\left(f_{2}\right)(x)
$$

Similar to $\mathrm{II}_{2}$, we have

$$
\begin{aligned}
& I V_{2} \leqslant C \frac{1}{|Q|} \int_{Q} \sum_{k=1}^{\infty}\left(\int_{S_{0}\left(Q^{*}\right) \times S_{k}\left(Q^{*}\right)}\left(\int_{0}^{\infty}\left|K_{t}\left(z, y_{1}, y_{2}\right)\right|^{2} \frac{d t}{t}\right)^{\frac{p_{0}^{\prime}}{2}} d y_{1} d y_{2}\right)^{1 / p_{0}^{\prime}} d z \\
& \times\left(\int_{Q^{*}}\left|\left(b_{1}\left(y_{1}\right)-\left(b_{1}\right)\right)_{Q^{*}}\right|^{p_{0}}\left|f_{1}\left(y_{1}\right)\right|^{p_{0}} d y_{1}\right)^{1 / p_{0}}\left(\int_{2^{k} Q^{*} \backslash 2^{k-1} Q^{*}}\left|\left(b_{2}\left(y_{2}\right)-\left(b_{2}\right)\right)_{Q^{*}}\right| p_{0}\left|f_{2}\left(y_{2}\right)\right|^{p_{0}} d y_{2}\right)^{1 / p_{0}} \\
& \leqslant C\left\|b_{1}\right\|_{\Lambda_{\beta_{1}}}\left\|b_{2}\right\|_{\lambda_{\beta_{2}}} \sum_{k=1}^{\infty} \frac{2^{-2 n k / p_{0}}}{\left|Q^{*}\right|^{\frac{2}{p_{0}}}}\left|Q^{*}\right|^{\frac{\beta_{2}}{n}}\left(\int_{2^{k} Q^{*}}\left|f_{1}\left(y_{1}\right)\right|^{p_{0}} d y_{1}\right)^{1 / p_{0}} \mid 2^{k} Q^{\frac{\beta_{2}}{n}}\left(\int_{Q^{*}}\left|f_{2}\left(y_{2}\right)\right|^{p_{0}} d y_{2}\right)^{1 / p_{0}}
\end{aligned}
$$

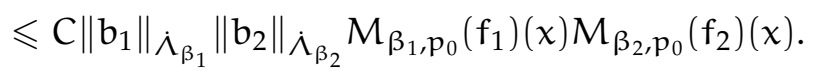

The same method to estimate $\mathrm{IV}_{2}$ says that

$$
I V_{3} \leqslant C\left\|b_{1}\right\|_{\dot{\Lambda}_{\beta_{1}}}\left\|b_{2}\right\|_{\dot{\Lambda}_{\beta_{2}}} M_{\beta_{1}, p_{0}}\left(f_{1}\right)(x) M_{\beta_{2}, p_{0}}\left(f_{2}\right)(x) .
$$

The condition $\left(\mathrm{H}_{2}\right)$ and Hölder's inequality yield that

$$
\begin{aligned}
& I V_{4} \leqslant C \frac{1}{|Q|} \int_{Q} \int_{R^{2 n}}\left(\int_{0}^{\infty}\left|K_{t}\left(z, y_{1}, y_{2}\right)-K_{t}\left(x_{Q}, y_{1}, y_{2}\right)\right|^{2} \frac{d t}{t}\right)^{\frac{1}{2}} \prod_{j=1}^{2}\left|b_{j}\left(y_{j}\right)-\left(b_{j}\right)_{Q^{*}} \| f_{j}^{\infty}\left(y_{j}\right)\right| d y_{1} d y_{2} d z \\
& \leqslant C \frac{1}{|Q|} \int_{Q_{j, k} \geqslant 1}\left(\int_{S_{j}\left(Q^{*}\right) \times S_{k}\left(Q^{*}\right)}\left(\left(\int_{0}^{\infty}\left|K_{t}\left(z, y_{1}, y_{2}\right)-K_{t}\left(x_{Q}, y_{1}, y_{2}\right)\right|^{2} \frac{d t}{t}\right)^{\frac{p_{0}^{\prime}}{2}} d y_{1} d y_{2}\right)^{\frac{1}{p_{0}^{\prime}}} d z\right. \\
& \times\left(\int_{2^{j} Q^{*}}\left|b_{1}\left(y_{1}\right)-\left(b_{1}\right)_{Q^{*}}\right|^{p_{0}}\left|f_{1}\left(y_{1}\right)\right|^{p_{0}} d y_{1}\right)^{1 / p_{0}}\left(\int_{2^{k} Q^{*}}\left|b_{2}\left(y_{2}\right)-\left(b_{2}\right)_{Q^{*}}\right|^{p_{0}}\left|f_{2}\left(y_{2}\right)\right|^{p_{0}} d y_{2}\right)^{1 / p_{0}}
\end{aligned}
$$

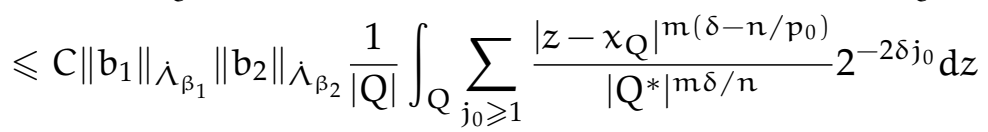

$$
\begin{aligned}
& \times\left|2^{j_{0}} Q^{*}\right|^{\frac{\beta_{1}}{n}}\left(\int_{2^{j_{0}} Q^{*}}\left|f_{1}\left(y_{1}\right)\right|^{p_{0}} d y_{1}\right)^{\frac{1}{p_{0}}}\left|2^{j_{0}} Q^{*}\right|^{\frac{\beta_{2}}{n}}\left(\int_{2^{j_{0}} Q^{*}}\left|f_{2}\left(y_{2}\right)\right|^{p_{0}} d y_{2}\right)^{\frac{1}{p_{0}}} \\
& \leqslant C\left\|b_{1}\right\|_{i_{\beta_{1}}}\left\|b_{2}\right\|_{i_{\beta_{2}}} \sum_{j_{0}>0} 2^{-2 j_{0}\left(\delta-\frac{\beta}{2}\right)} M_{\beta_{1}, p_{0}}\left(f_{1}\right)(x) M_{\beta_{2}, p_{0}}\left(f_{2}\right)(x) \\
& \leqslant C\left\|b_{1}\right\|_{{\dot{\lambda_{\beta}}}_{\beta_{1}}}\left\|b_{2}\right\|_{{\dot{\lambda}}_{\beta_{2}}} M_{\beta_{1}, p_{0}}\left(f_{1}\right)(x) M_{\beta_{2}, p_{0}}\left(f_{2}\right)(x) \text {. }
\end{aligned}
$$

From the estimates of I, II, III and IV, it follows that

$$
M_{\delta}^{\sharp}\left(T_{\Pi b}\left(f_{1}, f_{2}\right)\right)(x) \leqslant C\left\|b_{1}\right\|_{i_{\beta_{1}}}\left\|b_{2}\right\|_{\lambda_{\beta_{2}}}\left(M_{\beta}\left(T\left(f_{1}, f_{2}\right)(x)+M_{\beta_{1}, p_{0}}\left(f_{1}\right)(x) M_{\beta_{2}, p_{0}}\left(f_{2}\right)(x)\right),\right.
$$

which finishes the proof of this lemma. 
Now we are in position to prove Theorem 1.3.

Proof of Theorem 1.3. Thanks to Lemma 3.1 and the $\left(\mathrm{L}^{p_{1}} \times \cdots \times \mathrm{L}^{\mathrm{p}_{\mathrm{m}}}, \mathrm{L}^{\mathrm{p}}\right)$-boundedness of $\mathrm{T}$, the $\left(\mathrm{L}^{\mathrm{p}}, \mathrm{L}^{\mathrm{q}}\right)$ boundedness of fractional maximal operator $M_{\beta}, M_{\beta_{1}, p_{0}}$ and $M_{\beta_{2}, p_{0}}$,

$$
\begin{aligned}
\left\|T_{\Pi b}(\vec{f})\right\|_{L q} & \leqslant\left\|M_{\delta}\left(T_{\Pi b}(\vec{f})\right)\right\|_{L^{q}} \leqslant \| M_{\delta}^{\sharp}\left(T_{\Pi b}(\vec{f})(x) \|_{L^{q}}\right. \\
& \leqslant C \prod_{j=1}^{m}\left\|b_{j}\right\|_{\lambda_{\beta_{j}}}\left(\| M_{\beta}\left(T(\vec{f})\left\|_{L^{q}}+\right\| \prod_{j=1}^{m} M_{\beta_{j}, p_{0}}\left(f_{j}\right) \|_{L^{q}}\right)\right. \\
& \leqslant C \prod_{j=1}^{m}\left\|b_{j}\right\|_{\lambda_{\beta_{j}}}\left(\|T(\vec{f})\|_{L^{p}}+\prod_{j=1}^{m}\left\|M_{\beta_{j}, p_{0}}\left(f_{j}\right)\right\|_{L^{q_{j}}}\right) \\
& \leqslant C \prod_{j=1}^{m}\left\|b_{j}\right\|_{i_{\beta_{j}}}\left\|f_{j}\right\|_{L^{p_{j}}},
\end{aligned}
$$

which completes the proof of Theorem 1.3.

We are left to prove

$$
\left\|M_{\delta}\left(T_{\Pi b}(\vec{f})\right)\right\|_{L q}<\infty .
$$

The proof of (3.1) is standard, we refer the reader to [12].

\section{The commutators of multilinear square function on Lipschitz spaces}

In this section, we will show Theorem 1.4.

Proof of Theorem 1.4. For brief, we only need consider the case $m=2$ since the argument for $m>2$ is similar.

$$
\sup _{\mathrm{Q}} \frac{1}{|\mathrm{Q}|^{1+\frac{\beta}{n}-\frac{1}{p}}} \int_{Q}\left|T_{\Pi \mathbf{b}}\left(f_{1}, f_{2}\right)(z)-\left(T_{\Pi \mathbf{b}}\left(f_{1}, f_{2}\right)\right)_{Q}\right| d z \leqslant C \prod_{j=1}^{2}\left\|b_{j}\right\|_{\lambda_{\beta_{j}}}\left\|f_{j}\right\|_{L^{p_{j}}}
$$

Fix $Q=Q\left(x_{Q}, \ell_{Q}\right)$ which is any cube with center at $x_{Q}$ and side length $\ell_{Q}$. Let $\lambda_{i}=\left(b_{i}\right)_{Q^{*}}$ for $i=1,2$, and we split $f_{i}$ into the following form $f_{i}=f_{i} \chi_{Q^{*}}+f_{i} \chi_{R^{n}} \backslash Q^{*}=f_{i}^{0}+f_{i}^{\infty}$, where $Q^{*}=2 Q$.

We write

$$
\begin{aligned}
& \frac{1}{|\mathrm{Q}|^{1+\frac{\beta}{n}-\frac{1}{p}}} \int_{Q}\left|T_{\Pi b}\left(f_{1}, f_{2}\right)(z)-\left(T_{\Pi \mathbf{b}}\left(f_{1}, f_{2}\right)\right)_{Q}\right| d z \\
& \leqslant \mathrm{C} \frac{1}{|\mathrm{Q}|^{1+\frac{\beta}{n}-\frac{1}{p}}} \int_{\mathrm{Q}}\left|\mathrm{T}_{\Pi \mathbf{b}}\left(f_{1}^{0}, f_{2}^{0}\right)(z)\right| \mathrm{d} z+\frac{1}{|\mathrm{Q}|^{1+\frac{\beta}{n}-\frac{1}{p}}} \int_{\mathrm{Q}}\left|\mathrm{T}_{\Pi \mathbf{b}}\left(\mathrm{f}_{1}^{0}, \mathrm{f}_{2}^{\infty}\right)(z)\right| \mathrm{d} z \\
& +\frac{1}{|\mathrm{Q}|^{1+\frac{\beta}{n}-\frac{1}{p}}} \int_{\mathrm{Q}}\left|\mathrm{T}_{\Pi \mathbf{b}}\left(f_{1}^{\infty}, f_{2}^{0}\right)(z)\right| \mathrm{d} z+\frac{1}{|\mathrm{Q}|^{1+\frac{\beta}{n}-\frac{1}{p}}} \int_{\mathrm{Q}}\left|\mathrm{T}_{\Pi \mathbf{b}}\left(f_{1}^{\infty}, f_{2}^{\infty}\right)(z)-\mathrm{C}_{\mathrm{Q}}\right| \mathrm{d} z \\
& =A_{1}+A_{2}+A_{3}+A_{4} \text {, }
\end{aligned}
$$

where

$$
C_{Q}=\left(\int_{0}^{\infty}\left|\int_{R^{2 n}} K_{t}\left(x_{Q}, y_{1}, y_{2}\right) \prod_{j=1}^{2}\left(b_{j}\left(y_{j}\right)-\left(b_{j}\right)_{Q^{*}}\right) f_{j}^{\infty}\left(y_{j}\right) d y_{1} d y_{2}\right|^{2} \frac{d t}{t}\right)^{\frac{1}{2}} .
$$

We will estimate $A_{1}, A_{2}, A_{3}$ and $A_{4}$ respectively. Choose $1<q, p_{1}, p_{2}<\infty$ satisfying $\frac{1}{q}=\frac{1}{p_{1}}+\frac{1}{p_{2}}-\frac{\beta}{n}=\frac{1}{p}-\frac{\beta}{n}$. Then using Hölder's inequality and Theorem 1.3, one has

$$
A_{1} \leqslant C \frac{1}{|Q|^{1+\frac{\beta}{n}-\frac{1}{p}}}\left(\int_{Q}\left|T_{\Pi b}\left(f_{1}^{0}, f_{2}^{0}\right)(z)\right|^{q} d z\right)^{\frac{1}{q}}|Q|^{1-\frac{1}{q}}
$$




$$
\leqslant C \prod_{j=1}^{m}\left\|b_{j}\right\|_{\lambda_{\beta_{j}}}\left\|f_{j}\right\|_{L^{p_{j}\left(R^{n}\right)}} .
$$

For the second term $A_{2}$, we split $A_{2}$ into the following form:

$$
\begin{aligned}
A_{2} \leqslant & C \frac{1}{|Q|^{1+\frac{\beta}{n}-\frac{1}{p}}} \int_{Q}\left|b_{1}(z)-\left(b_{1}\right)_{Q^{*}} \| b_{2}(z)-\left(b_{2}\right)_{Q^{*}}\right| T\left(f_{1}^{\infty}, f_{2}^{0}\right)(z) \mid d z \\
& +C \frac{1}{\mid Q^{1+\frac{\beta}{n}-\frac{1}{p}}} \int_{Q}\left|b_{1}(z)-\left(b_{1}\right)_{Q^{*}}\right| T\left(f_{1}^{\infty},\left(b_{2}-\left(b_{2}\right)_{Q^{*}}\right) f_{2}^{0}\right)(z) \mid d z \\
& +C \frac{1}{|Q|^{1+\frac{\beta}{n}-\frac{1}{p}}} \int_{Q}\left|\left(b_{2}-\left(b_{2}\right)_{Q^{*}}\right)\right| T\left(\left(b_{1}(z)-\left(b_{1}\right)_{Q^{*}}\right) f_{1}^{\infty}, f_{2}^{0}\right)(z) \mid d z \\
& +C \frac{1}{\mid Q^{1+\frac{\beta}{n}-\frac{1}{p}}} \int_{Q}\left|T\left(\left(b_{1}(z)-\left(b_{1}\right)_{Q^{*}}\right) f_{1}^{\infty},\left(b_{2}-\left(b_{2}\right)_{Q^{*}}\right) f_{2}^{0}\right)(z)\right| d z \\
= & A_{21}+A_{22}+A_{23}+A_{24} .
\end{aligned}
$$

Condition $\left(\mathrm{H}_{3}\right)$ in Definition 1.1 and Hölder's inequality give that

$$
\begin{aligned}
& A_{21} \leqslant C \prod_{j=1}^{2}\left\|b_{j}\right\|_{i_{\beta_{j}}} \frac{1}{|Q|^{1-\frac{1}{p}}} \int_{Q} \int_{R^{2}}\left(\int_{0}^{\infty}\left|K_{t}\left(x, y_{1}, y_{2}\right)\right|^{2} \frac{d t}{t}\right)^{\frac{1}{2}}\left|f_{1}^{\infty}\left(y_{1}\right) \| f_{2}^{0}\left(y_{2}\right)\right| d y_{1} d y_{2} d z \\
& \leqslant C \prod_{j=1}^{2}\left\|b_{j}\right\|_{\lambda_{\beta_{j}}} \frac{1}{|Q|^{1-\frac{1}{p}}} \int_{Q} \sum_{k=1}^{\infty}\left(\int_{S_{0}\left(Q^{*}\right) \times S_{k}\left(Q^{*}\right)}\left(\int_{0}^{\infty}\left|K_{t}\left(z, y_{1}, y_{2}\right)\right|^{2} \frac{d t}{t}\right)^{\frac{p_{0}^{\prime}}{2}} d y_{1} d y_{2}\right)^{1 / p_{0}^{\prime}} d z \\
& \times\left(\int_{2^{k} Q^{*} \backslash 2^{k-1} Q^{*}}\left|f_{1}\left(y_{1}\right)\right|^{p_{0}} d y_{1}\right)^{1 / p_{0}}\left(\int_{Q^{*}}\left|f_{2}\left(y_{2}\right)\right|^{p_{0}} d y_{2}\right)^{1 / p_{0}} d z \\
& \leqslant\left. C \prod_{j=1}^{2}\left\|b_{j}\right\|_{\lambda_{\beta_{j}}}\left|Q^{\frac{1}{p}} \sum_{k=1}^{\infty} \frac{2^{-2 n k / p_{0}}}{\left|Q^{*}\right|^{\frac{2}{p_{0}}}}\left\|f_{1}\right\|_{L^{p_{1}}}\left\|f_{2}\right\|_{L^{p_{2}}}\right|^{k} Q^{*}\right|^{\frac{1}{p_{0}}}-\frac{1}{p_{1}}\left|Q^{*}\right|^{\frac{1}{p_{0}}}-\frac{1}{p_{2}} \\
& \leqslant C \prod_{j=1}^{2}\left\|b_{j}\right\|_{{\dot{\beta_{\beta}}}_{j}}\left\|f_{j}\right\|_{L^{p_{j}\left(R^{n}\right)}}
\end{aligned}
$$

Since $A_{22}$ and $A_{23}$ are very similar, we only need to consider the second term $A_{22}$.

$$
\begin{aligned}
& A_{22} \leqslant C\left\|b_{1}\right\|_{\lambda_{\beta_{1}}} \frac{1}{|Q|^{1+\frac{\beta_{2}}{n}-\frac{1}{p}}} \int_{Q} \int_{R^{2 n}}\left(\int_{0}^{\infty}\left|K_{t}\left(z, y_{1}, y_{2}\right)\right|^{2} \frac{d t}{t}\right)^{\frac{1}{2}} \\
& \times\left|f_{1}^{\infty}\left(y_{1}\right)\right|\left|\left(b_{2}\left(y_{2}\right)-\left(b_{2}\right)\right) Q^{*} \| f_{2}^{0}\left(y_{2}\right)\right| d y_{1} d y_{2} d z \\
& \leqslant C\left\|b_{1}\right\|_{\dot{\lambda}_{\beta_{1}}}|\mathrm{Q}|^{\frac{1}{p}-\frac{\beta_{2}}{n}-1} \int_{Q_{k=1}}^{\infty}\left(\int_{S_{0}\left(Q^{*}\right) \times S_{k}\left(Q^{*}\right)}\left(\int_{0}^{\infty}\left|K_{t}\left(z, y_{1}, y_{2}\right)\right|^{2} \frac{d t}{t}\right)^{\frac{p_{0}^{\prime}}{2}} d y_{1} d y_{2}\right)^{1 / p_{0}^{\prime}} d z \\
& \times\left(\int_{2^{k} Q^{*} \backslash 2^{k-1} Q^{*}}\left|f_{1}\left(y_{1}\right)\right|^{p_{0}} d y_{1}\right)^{1 / p_{0}}\left(\left.\int_{Q^{*}}\left|\left(b_{2}\left(y_{2}\right)-\left(b_{2}\right)\right)_{Q^{*}} p^{p_{0}}\right| f_{2}\left(y_{2}\right)\right|^{p_{0}} d y_{2}\right)^{1 / p_{0}} \\
& \leqslant C\left\|b_{1}\right\|_{\dot{\Lambda}_{\beta_{1}}}\left\|b_{2}\right\|_{{\dot{\beta_{\beta}}}_{\beta_{2}}} \mid Q^{\frac{1}{p}} \sum_{k=1}^{\infty} \frac{2^{-2 n k / p_{0}}}{\left|Q^{*}\right| \frac{2}{p_{0}}}\left(\int_{2^{k} Q^{*}}\left|f_{1}\left(y_{1}\right)\right|^{p_{0}} d y_{1}\right)^{1 / p_{0}}\left(\int_{Q^{*}}\left|f_{2}\left(y_{2}\right)\right|^{p_{0}} d y_{2}\right)^{1 / p_{0}} \\
& \leqslant\left. C \prod_{j=1}^{2}\left\|b_{j}\right\|_{\lambda_{\beta_{j}}}\left|Q^{\frac{1}{p}} \sum_{k=1}^{\infty} \frac{2^{-2 n k / p_{0}}}{\left|Q^{*}\right|^{\frac{2}{p_{0}}}}\left\|f_{1}\right\|_{L^{p_{1}}}\left\|f_{2}\right\|_{L^{p_{2}}}\right| 2^{k} Q^{*}\right|^{\frac{1}{p_{0}}}-\frac{1}{p_{1}}\left|Q^{*}\right|^{\frac{1}{p_{0}}}-\frac{1}{p_{2}}
\end{aligned}
$$




$$
\leqslant C \prod_{j=1}^{2}\left\|b_{j}\right\|_{i_{\beta_{j}}}\left\|f_{j}\right\|_{L^{p_{j}\left(R^{n}\right)}}
$$

Similarly, we also have

$$
A_{23} \leqslant C \prod_{j=1}^{2}\left\|b_{j}\right\|_{\Lambda_{\beta_{j}}}\left\|f_{j}\right\|_{L^{p_{j}\left(R^{n}\right)}} .
$$

For the last term $A_{24}$, thanks to condition $\left(\mathrm{H}_{3}\right)$ and the Hölder inequality, we have

$$
\begin{aligned}
& A_{24} \leqslant \frac{1}{|Q|^{1+\frac{\beta}{n}-\frac{1}{p}}} \int_{Q} \int_{R^{2 n}}\left(\int_{0}^{\infty}\left|K_{t}\left(z, y_{1}, y_{2}\right)\right|^{2} \frac{d t}{t}\right)^{\frac{1}{2}}\left|\left(b_{1}\left(y_{1}\right)-\left(b_{1}\right)\right)_{Q^{*}}\right| \\
& \times\left|f_{1}^{\infty}\left(y_{1}\right)\left\|\left(b_{2}\left(y_{2}\right)-\left(b_{2}\right)\right) Q^{*}\right\| f_{2}^{0}\left(y_{2}\right)\right| d y_{1} d y_{2} d z \\
& \leqslant C \frac{1}{|Q|^{1+\frac{\beta}{n}-\frac{1}{p}}} \int_{Q} \sum_{k=1}^{\infty}\left(\int_{S_{0}\left(Q^{*}\right) \times S_{k}\left(Q^{*}\right)}\left(\int_{0}^{\infty}\left|K_{t}\left(z, y_{1}, y_{2}\right)\right|^{2} \frac{d t}{t}\right)^{\frac{p_{0}^{\prime}}{2}} d y_{1} d y_{2}\right)^{1 / p_{0}^{\prime}} d z \\
& \times\left(\left.\int_{2^{k} Q^{*} \backslash 2^{k-1} Q^{*}}\left|\left(b_{1}\left(y_{1}\right)-\left(b_{1}\right)\right)_{Q^{*}} p^{p_{0}}\right| f_{1}\left(y_{1}\right)\right|^{p_{0}} d y_{1}\right)^{1 / p_{0}} \\
& \times\left(\int_{\mathrm{Q}^{*}}\left|\left(\mathrm{~b}_{2}\left(\mathrm{y}_{2}\right)-\left(\mathrm{b}_{2}\right)\right)_{\mathrm{Q}^{*}}\right|^{\mathrm{p}_{0}}\left|\mathrm{f}_{2}\left(\mathrm{y}_{2}\right)\right|^{\mathrm{p}_{0}} \mathrm{~d} \mathrm{y}_{2}\right)^{1 / \mathrm{p}_{0}} \\
& \leqslant\left. C \prod_{j=1}^{2}\left\|b_{j}\right\|_{\Lambda_{\beta_{j}}}\left|Q^{\frac{1}{p}} \sum_{k=1}^{\infty} \frac{2^{-2 n k / p_{0}}}{\left|Q^{*}\right|^{\frac{2}{p_{0}}}}\left\|f_{1}\right\|_{L^{p_{1}}}\left\|f_{2}\right\|_{L^{p_{2}}}\right| 2^{k} Q^{*}\right|^{\frac{1}{p_{0}}-\frac{1}{p_{1}}}\left|Q^{*}\right|^{\frac{1}{p_{0}}-\frac{1}{p_{2}}} \\
& \leqslant C \prod_{j=1}^{2}\left\|b_{j}\right\|_{i_{\beta_{j}}}\left\|f_{j}\right\|_{L^{p_{j}\left(R^{n}\right)}} \text {. }
\end{aligned}
$$

Finally, we bound the fourth term $A_{4}$. Similar to $A_{2}$, we also need to split $A_{4}$ into the following four terms.

$$
\begin{aligned}
A_{4} \leqslant & C \frac{1}{|Q|^{1+\frac{\beta}{n}-\frac{1}{p}}} \int_{Q}\left|T\left(\left(b_{1}-\left(b_{1}\right)_{Q^{*}}\right) f_{1}^{0},\left(b_{2}-\left(b_{2}\right)_{Q^{*}}\right) f_{2}^{0}\right)(z)\right| d z \\
& +C \frac{1}{|Q|^{1+\frac{\beta}{n}-\frac{1}{p}}} \int_{Q}\left|T\left(\left(b_{1}-\left(b_{1}\right)_{Q^{*}}\right) f_{1}^{0},\left(b_{2}-\left(b_{2}\right)_{Q^{*}}\right) f_{2}^{\infty}\right)(z)\right| d z \\
& +C \frac{1}{|Q|^{1+\frac{\beta}{n}-\frac{1}{p}}} \int_{Q}\left|T\left(\left(b_{1}-\left(b_{1}\right)_{Q^{*}}\right) f_{1}^{\infty},\left(b_{2}-\left(b_{2}\right)_{Q^{*}}\right) f_{2}^{0}\right)(z)\right| d z \\
& +C \frac{1}{|Q|^{1+\frac{\beta}{n}-\frac{1}{p}}} \int_{Q}\left|T\left(\left(b_{1}-\left(b_{1}\right)_{Q^{*}}\right) f_{1}^{\infty},\left(b_{2}-\left(b_{2}\right)_{Q^{*}}\right) f_{2}^{\infty}\right)(z)-C_{Q}\right| d z \\
= & A_{41}+A_{42}+A_{43}+A_{44} .
\end{aligned}
$$

Following the same line to bound $A_{1}$, we have

$$
A_{41} \leqslant C \prod_{j=1}^{2}\left\|b_{j}\right\|_{i_{\beta_{j}}}\left\|f_{j}\right\|_{L^{p_{j}}} .
$$

Due to $A_{42}$ and $A_{43}$ are similar, we only handle the second term $A_{42}$. Similar to $A_{22}$, it is easy to follow

$$
A_{42} \leqslant C \frac{1}{|Q|^{1+\frac{\beta}{n}-\frac{1}{p}}} \int_{Q} \sum_{k=1}^{\infty}\left(\int_{S_{k}\left(Q^{*}\right) \times S_{0}\left(Q^{*}\right)}\left(\int_{0}^{\infty}\left|K_{t}\left(z, y_{1}, y_{2}\right)\right|^{2} \frac{d t}{t}\right)^{\frac{p_{0}^{\prime}}{2}} d y_{1} d y_{2}\right)^{1 / p_{0}^{\prime}} d z
$$




$$
\begin{aligned}
& \times\left(\int_{2^{k} Q^{*} \backslash 2^{k-1} Q^{*}}\left|\left(b_{1}\left(y_{1}\right)-\left(b_{1}\right)\right)_{Q^{*}}\right|^{p_{0}}\left|f_{1}\left(y_{1}\right)\right|^{p_{0}} d y_{1}\right)^{1 / p_{0}} \\
& \times\left(\int_{Q^{*}}\left|\left(b_{2}\left(y_{2}\right)-\left(b_{2}\right)\right)_{Q^{*}}\right| p_{0}\left|f_{2}\left(y_{2}\right)\right|^{p_{0}} d y_{2}\right)^{1 / p_{0}} \\
\leqslant & \left.|Q|^{-\frac{\beta}{n}+\frac{1}{p}} \sum_{k=1}^{\infty} \frac{2^{-2 k n / p_{0}}}{\left|Q^{*}\right|^{\frac{2}{p_{0}}}}\left|2^{k} Q^{*} \frac{\beta_{1}}{n}\left\|b_{1}\right\|_{\lambda_{\beta_{1}}}\right| 2^{k} Q^{*}\left|\frac{1}{p_{0}}-\frac{1}{p_{1}}\left\|f_{1}\right\|_{L^{p_{1}}}\right| Q^{*}\right|^{\frac{\beta_{2}}{n}}\left\|b_{2}\right\|_{\lambda_{\beta_{2}}}\left|Q^{*}\right| \frac{1}{p_{0}}-\frac{1}{p_{2}}\left\|f_{2}\right\|_{L^{p_{2}}} \\
\leqslant & C \prod_{j=1}^{2}\left\|b_{j}\right\|_{\lambda_{\beta_{j}}}\left\|f_{j}\right\|_{L^{p_{j}}} \sum_{k=1}^{\infty} 2^{-k n\left(\frac{\beta_{1}}{n}-\frac{1}{p_{1}}\right)} \leqslant C \prod_{j=1}^{2}\left\|b_{j}\right\|_{\dot{\Lambda}_{\beta_{j}}}\left\|f_{j}\right\|_{L^{p_{j}},}
\end{aligned}
$$

here we have used the fact that $p_{1}>\frac{n}{\beta_{1}}$.

Similarly, we have

$$
A_{43} \leqslant C \prod_{j=1}^{2}\left\|b_{j}\right\|_{\dot{\Lambda}_{\beta_{j}}}\left\|f_{j}\right\|_{L^{p_{j}}}
$$

for $p_{2}>\frac{n}{\beta_{2}}$.

At last we shall handle the fourth term $A_{44}$. Similar to $A_{24}$, the condition $\left(\mathrm{H}_{2}\right)$ and Hölder's inequality yield that

$$
\begin{aligned}
& A_{44} \leqslant C \frac{1}{|Q|^{1+\frac{\beta}{n}-\frac{1}{p}}} \int_{Q} \int_{R^{2 n}}\left(\int_{0}^{\infty}\left|K_{t}\left(z, y_{1}, y_{2}\right)-K_{t}\left(x_{Q}, y_{1}, y_{2}\right)\right|^{2} \frac{d t}{t}\right)^{\frac{1}{2}} \\
& \times \prod_{j=1}^{2}\left|b_{j}\left(y_{j}\right)-\left(b_{j}\right)_{Q^{*}} \| f_{j}^{\infty}\left(y_{j}\right)\right| d y_{1} d y_{2} d z \\
& \left.\leqslant C \frac{1}{|Q|^{1+\frac{\beta}{n}-\frac{1}{p}}} \int_{Q_{j, k \geqslant 1}} \sum_{S_{j}\left(Q^{*}\right) \times S_{k}\left(Q^{*}\right)}\left(\int_{0}^{\infty}\left|K_{t}\left(z, y_{1}, y_{2}\right)-K_{t}\left(x_{Q}, y_{1}, y_{2}\right)\right|^{2} \frac{d t}{t}\right)^{\frac{p_{0}^{\prime}}{2}} d y_{1} d y_{2}\right)^{\frac{1}{p_{0}^{\prime}}} d z \\
& \times\left(\int_{2^{j} Q^{*}}\left|b_{1}\left(y_{1}\right)-\left(b_{1}\right)_{Q^{*}}\right|^{p_{0}}\left|f_{1}\left(y_{1}\right)\right|^{p_{0}} d y_{1}\right)^{1 / p_{0}}\left(\int_{2^{k} Q^{*}}\left|b_{2}\left(y_{2}\right)-\left(b_{2}\right)_{Q^{*}}\right|^{p_{0}}\left|f_{2}\left(y_{2}\right)\right|^{p_{0}} d y_{2}\right)^{1 / p_{0}} \\
& \leqslant C\left\|b_{1}\right\|_{\lambda_{\beta_{1}}}\left\|b_{2}\right\|_{i_{\beta_{2}}} \frac{1}{|Q|^{1+\frac{\beta}{n}-\frac{1}{p}}} \sum_{j_{0} \geqslant 1} \int_{Q} \frac{\left|z-x_{Q}\right|^{\left(\delta-n / p_{0}\right)}}{\left|Q^{*}\right|^{2 \delta / n}} 2^{-2 \delta j_{0}} d z \\
& \times\left|2^{j_{0}} Q^{*}\right|^{\frac{\beta_{1}}{n}}\left(\int_{2^{j}{ }_{0} Q^{*}}\left|f_{1}\left(y_{1}\right)\right|^{p_{0}} d y_{1}\right)^{\frac{1}{p_{0}}}\left|2^{j_{0}} Q^{*}\right|^{\frac{\beta_{2}}{n}}\left(\int_{2^{j} Q^{*}}\left|f_{2}\left(y_{2}\right)\right|^{p_{0}} d y_{2}\right)^{\frac{1}{p_{0}}} \\
& \leqslant\left. C\left\|b_{1}\right\|_{\lambda_{\beta_{1}}}\left\|b_{2}\right\|_{\dot{\lambda}_{\beta_{2}}}\left|Q^{\frac{1}{p}-\frac{\beta}{n}} \sum_{j_{0} \geqslant 1} 2^{-j_{0}(2 \delta-\beta)}\right| Q^{*}\left|\frac{\beta_{1}}{n}+\frac{\beta_{2}}{n}\left\|f_{1}\right\|_{L^{p_{1}}}\right| 2^{j_{0}} Q^{*}\left|\frac{1}{p_{0}}-\frac{1}{p_{1}}\left\|f_{2}\right\|_{L^{p_{2}}}\right| 2^{j_{0}} Q^{*}\right|^{\frac{1}{p_{0}}-\frac{1}{p_{2}}} \\
& \leqslant C \prod_{j=1}^{2}\left\|b_{j}\right\|_{\lambda_{\beta_{j}}}\left\|f_{j}\right\|_{L^{p_{j}}} \sum_{j_{0} \geqslant 1} 2^{-2 j_{0}\left(\delta-\beta / 2-n\left(\frac{1}{p_{0}}-\frac{1}{2 p}\right)\right)} \leqslant C \prod_{j=1}^{2}\left\|b_{j}\right\|_{{\dot{\beta_{\beta}}}_{j}}\left\|f_{j}\right\|_{L^{p_{j}}},
\end{aligned}
$$

where we have used the condition $\delta>\frac{\beta}{2}-\mathfrak{n}\left(\frac{1}{p_{0}}-\frac{1}{2 p}\right)$.

\section{Commutators of multilinear square functions on BMO spaces}

In this section, we will prove the boundedness of commutators of multilinear square function on BMO spaces. 
Proof. For simplicity, we only consider the case $m=2$, the other case $m>2$ are very similar. We write

$$
\begin{aligned}
& \frac{1}{|Q|} \int_{Q}\left|T_{\Pi \mathbf{b}}\left(f_{1}, f_{2}\right)(z)-\left(T_{\Pi \mathbf{b}}\left(f_{1}, f_{2}\right)\right)_{Q}\right| d z \\
& \leqslant C \frac{1}{|Q|} \int_{Q}\left|T_{\Pi \mathbf{b}}\left(f_{1}^{0}, f_{2}^{0}\right)(z)\right| d z+\frac{1}{|Q|} \int_{Q}\left|T_{\Pi \mathbf{b}}\left(f_{1}^{0}, f_{2}^{\infty}\right)(z)\right| d z \\
&+\frac{1}{|Q|} \int_{Q}\left|T_{\Pi \mathbf{b}}\left(f_{1}^{\infty}, f_{2}^{0}\right)(z)\right| d z+\frac{1}{|Q|} \int_{Q}\left|T_{\Pi b}\left(f_{1}^{\infty}, f_{2}^{\infty}\right)(z)-C_{Q}\right| d z \\
&= B_{1}+B_{2}+B_{3}+B_{4},
\end{aligned}
$$

where

$$
C_{Q}=\left(\int_{0}^{\infty}\left|\int_{R^{2 n}} K_{t}\left(x_{Q}, y_{1}, y_{2}\right) \prod_{j=1}^{2}\left(b_{j}\left(y_{j}\right)-\left(b_{j}\right)_{Q^{*}}\right) f_{j}^{\infty}\left(y_{j}\right) d y_{1} d y_{2}\right|^{2} \frac{d t}{t}\right)^{\frac{1}{2}} .
$$

Now we cope with $B_{1}, B_{2}, B_{3}$ and $B_{4}$, respectively. To bound $B_{1}$, we write

$$
\begin{aligned}
\mathrm{B}_{1} \leqslant & \mathrm{C} \frac{1}{|\mathrm{Q}|} \int_{\mathrm{Q}}\left|\mathrm{b}_{1}(z)-\left(\mathrm{b}_{1}\right)_{\mathrm{Q}^{*}}\right|\left|\mathrm{b}_{2}(z)-\left(\mathrm{b}_{2}\right)_{\mathrm{Q}^{*}}\right|\left|\mathrm{T}\left(\mathrm{f}_{1}^{0}, \mathrm{f}_{2}^{0}\right)(z)\right| \mathrm{d} z \\
& +\mathrm{C} \frac{1}{|\mathrm{Q}|} \int_{\mathrm{Q}}\left|\mathrm{b}_{1}(z)-\left(\mathrm{b}_{1}\right)_{\mathrm{Q}^{*}} \| \mathrm{T}\left(\mathrm{f}_{1}^{0},\left(\mathrm{~b}_{2}-\left(\mathrm{b}_{2}\right)_{\mathrm{Q}^{*}}\right) \mathrm{f}_{2}^{0}\right)(z)\right| \mathrm{d} z \\
& +\mathrm{C} \frac{1}{|\mathrm{Q}|} \int_{\mathrm{Q}}\left|\mathrm{b}_{2}(z)-\left(\mathrm{b}_{2}\right)_{\mathrm{Q}^{*}}\right|\left|\mathrm{T}\left(\left(\mathrm{b}_{1}-\left(\mathrm{b}_{1}\right)_{\mathrm{Q}^{*}}\right) \mathrm{f}_{1}^{0}, \mathrm{f}_{2}^{0}\right)(z)\right| \mathrm{d} z \\
& +\mathrm{C} \frac{1}{|\mathrm{Q}|} \int_{\mathrm{Q}}\left|\mathrm{T}\left(\left(\mathrm{b}_{1}-\left(\mathrm{b}_{1}\right)_{\mathrm{Q}^{*}}\right) \mathrm{f}_{1}^{0},\left(\mathrm{~b}_{2}-\left(\mathrm{b}_{2}\right)_{\mathrm{Q}^{*}}\right) \mathrm{f}_{2}^{0}\right)(z)\right| \mathrm{d} z \\
= & \mathrm{B}_{11}+\mathrm{B}_{12}+\mathrm{B}_{13}+\mathrm{B}_{14} .
\end{aligned}
$$

To estimate $\mathrm{B}_{11}$ : The condition $\left(\mathrm{H}_{4}\right)$ and Hölder's inequality say that

$$
\begin{aligned}
& B_{11} \leqslant C \frac{1}{|Q|} \int_{Q}\left(\int_{S_{0}\left(Q^{*}\right) \times S_{0}\left(Q^{*}\right)}\left(\int_{0}^{\infty}\left|K_{t}\left(z, y_{1}, y_{2}\right)\right|^{2} \frac{d t}{t}\right)^{\frac{p_{0}}{2}} d y_{1} d y_{2}\right)^{1 / p_{0}} d z \\
& \times \prod_{j=1}^{2}\left\|b_{j}\right\|_{i_{\beta_{j}}}\left|Q^{*}\right|^{\frac{\beta}{n}} \prod_{j=1}^{2}\left(\int_{Q^{*}}\left|f_{j}\left(y_{j}\right)\right|^{p_{0}} d y_{j}\right)^{\frac{1}{p_{0}}} \\
& \leqslant C \prod_{j=1}^{2}\left\|b_{j}\right\|_{\dot{\lambda}_{\beta_{j}}}\left|Q^{*}\right|^{\frac{\beta}{n}}\left|Q^{*}\right|^{-\frac{2}{p_{0}}}\left\|f_{1}\right\|_{L^{\frac{n}{\beta_{1}}}}\left\|f_{2}\right\|_{L^{\frac{n}{\beta_{2}}}}\left|Q^{*}\right|^{\frac{2}{p_{0}}-\frac{\beta}{n}} \\
& \leqslant C \prod_{j=1}^{2}\left\|b_{j}\right\|_{\lambda_{\beta_{j}}}\left\|f_{1}\right\|_{L^{\frac{n}{\beta_{1}}}}\left\|f_{2}\right\|_{L^{\frac{n}{\beta_{2}}}}
\end{aligned}
$$

where we used the assumption $p_{0}<\min \left\{\frac{n}{\beta_{1}}, \frac{n}{\beta_{2}}\right\}$.

Following the same argument to $B_{11}$, it is easy for us to prove

$$
B_{1 i} \leqslant C \prod_{j=1}^{2}\left\|b_{j}\right\|_{\Lambda_{\beta_{j}}}\left\|f_{1}\right\|_{L^{\frac{n}{\beta_{1}}}}\left\|f_{2}\right\|_{L^{\frac{n}{\beta_{2}}}}
$$

for $i=2,3,4$, which gives that

$$
B_{1} \leqslant C \prod_{j=1}^{2}\left\|b_{j}\right\|_{\dot{\Lambda}_{\beta_{j}}}\left\|f_{1}\right\|_{L^{\frac{n}{\beta_{1}}}}\left\|f_{2}\right\|_{L^{\frac{n}{\beta_{2}}}}
$$


For the second term $B_{2}$, we also split it into the following form:

$$
\begin{aligned}
\mathrm{B}_{2} \leqslant & \mathrm{C} \frac{1}{|\mathrm{Q}|} \int_{\mathrm{Q}}\left|\mathrm{b}_{1}(z)-\left(\mathrm{b}_{1}\right)_{\mathrm{Q}^{*}}\left\|\mathrm{~b}_{2}(z)-\left(\mathrm{b}_{2}\right)_{\mathrm{Q}^{*}}\right\| \mathrm{T}\left(\mathrm{f}_{1}^{0}, \mathrm{f}_{2}^{\infty}\right)(z)\right| \mathrm{d} z \\
& +\mathrm{C} \frac{1}{|\mathrm{Q}|} \int_{\mathrm{Q}}\left|\mathrm{b}_{1}(z)-\left(\mathrm{b}_{1}\right)_{\mathrm{Q}^{*}} \| \mathrm{T}\left(\mathrm{f}_{1}^{0},\left(\mathrm{~b}_{2}-\left(\mathrm{b}_{2}\right)_{\mathrm{Q}^{*}}\right) \mathrm{f}_{2}^{\infty}\right)(z)\right| \mathrm{d} z \\
& +\mathrm{C} \frac{1}{|\mathrm{Q}|} \int_{\mathrm{Q}}\left|\mathrm{b}_{2}(z)-\left(\mathrm{b}_{2}\right)_{\mathrm{Q}^{*}} \| \mathrm{T}\left(\left(\mathrm{b}_{1}-\left(\mathrm{b}_{1}\right)_{\mathrm{Q}^{*}}\right) \mathrm{f}_{1}^{0}, \mathrm{f}_{2}^{\infty}\right)(z)\right| \mathrm{d} z \\
& +\mathrm{C} \frac{1}{|\mathrm{Q}|} \int_{\mathrm{Q}}\left|\mathrm{T}\left(\left(\mathrm{b}_{1}-\left(\mathrm{b}_{1}\right)_{\mathrm{Q}^{*}}\right) \mathrm{f}_{1}^{0},\left(\mathrm{~b}_{2}-\left(\mathrm{b}_{2}\right)_{\mathrm{Q}^{*}}\right) \mathrm{f}_{2}^{\infty}\right)(z)\right| \mathrm{d} z \\
= & \mathrm{B}_{21}+\mathrm{B}_{22}+\mathrm{B}_{23}+\mathrm{B}_{24} .
\end{aligned}
$$

The condition $\left(\mathrm{H}_{3}\right)$ and Hölder's inequality yield that

$$
\begin{aligned}
& \mathrm{B}_{21} \leqslant \mathrm{C} \sum_{\mathrm{k}=1}^{\infty} \frac{1}{|\mathrm{Q}|} \int_{\mathrm{Q}}\left(\int_{\mathrm{S}_{0}\left(\mathrm{Q}^{*}\right) \times \mathrm{S}_{\mathrm{k}}\left(\mathrm{Q}^{*}\right)}\left(\int_{0}^{\infty}\left|\mathrm{K}_{\mathrm{t}}\left(z, \mathrm{y}_{1}, \mathrm{y}_{2}\right)\right|^{2} \frac{\mathrm{dt}}{\mathrm{t}}\right)^{\frac{\mathrm{p}_{0}}{2}} \mathrm{~d} \mathrm{y}_{1} \mathrm{~d} \mathrm{y}_{2}\right)^{1 / \mathrm{p}_{0}} \mathrm{~d} z \\
& \times \prod_{j=1}^{2}\left\|b_{j}\right\|_{\lambda_{\beta_{j}}}\left|Q^{*}\right| \frac{\beta}{n}\left(\int_{Q^{*}}\left|f_{1}\left(y_{1}\right)\right|^{p_{0}} d y_{1}\right)^{\frac{1}{p_{0}}}\left(\int_{2^{k} Q^{*}}\left|f_{2}\left(y_{2}\right)\right|^{p_{0}} d y_{2}\right)^{\frac{1}{p_{0}}} \\
& \leqslant C \prod_{j=1}^{2}\left\|b_{j}\right\|_{\dot{\Lambda}_{\beta_{j}}}\left|Q^{*}\right|^{\frac{\beta}{n}} \sum_{k=1}^{\infty} \frac{1}{|Q|} \int_{Q} \frac{2^{-2 k n / p_{0}}}{\left|Q^{*}\right|^{\frac{2}{p_{0}}}} d z\left|Q^{*}\right|^{\frac{1}{p_{0}}-\frac{\beta_{1}}{n}}\left\|f_{1}\right\|_{L^{\frac{n}{\beta_{1}}}}\left\|f_{2}\right\|_{L^{\frac{n}{\beta_{2}}}}\left|2^{k} Q^{*}\right|^{\frac{1}{p_{0}}-\frac{\beta_{2}}{n}} \\
& \leqslant C \prod_{j=1}^{2}\left\|b_{j}\right\|_{\Lambda_{\beta_{j}}}\left\|f_{1}\right\|_{L^{\frac{n}{\beta_{1}}}}\left\|f_{2}\right\|_{L^{\frac{n}{\beta_{2}}}} \sum_{k=1}^{\infty} 2^{-k n\left(-\frac{1}{p_{0}}+\frac{\beta_{2}}{n}\right)} \\
& \leqslant C \prod_{j=1}^{2}\left\|b_{j}\right\|_{\Lambda_{\beta_{j}}}\left\|f_{1}\right\|_{L^{\frac{n}{\beta_{1}}}}\left\|f_{2}\right\|_{L^{\frac{n}{\beta_{2}}}}
\end{aligned}
$$

because of the fact that $p_{0}<\frac{n}{\beta_{2}}$.

The same argument to bound $\mathrm{B}_{12}, \mathrm{~B}_{13}$ and $\mathrm{B}_{14}$ yields that

$$
B_{2 i} \leqslant C \prod_{j=1}^{2}\left\|b_{j}\right\|_{\Lambda_{\beta_{j}}}\left\|f_{1}\right\|_{L^{\frac{n}{\beta_{1}}}}\left\|f_{2}\right\|_{L^{\frac{n}{\beta_{2}}}}
$$

for $i=2,3,4$, which in turn imply that

$$
\mathrm{B}_{2} \leqslant \mathrm{C} \prod_{j=1}^{2}\left\|\mathrm{~b}_{j}\right\|_{{\dot{\lambda}}_{\beta_{j}}}\left\|f_{1}\right\|_{L^{\frac{n}{\beta_{1}}}}\left\|f_{2}\right\|_{L^{\frac{n}{\beta_{2}}}} .
$$

Similarly, it also has

$$
B_{3} \leqslant C \prod_{j=1}^{2}\left\|b_{j}\right\|_{\lambda_{\beta_{j}}}\left|Q^{*}\right|^{\frac{\beta}{n}}\left\|f_{1}\right\|_{L^{\frac{n}{\beta_{1}}}}\left\|f_{2}\right\|_{L^{\frac{n}{\beta_{2}}}} .
$$

Finally, we will handle the fourth term $B_{4}$. We first need decompose $B_{4}$ into the following terms:

$$
\mathrm{B}_{4} \leqslant \mathrm{C} \frac{1}{|\mathrm{Q}|} \int_{\mathrm{Q}}\left|\mathrm{b}_{1}(z)-\left(\mathrm{b}_{1}\right)_{\mathrm{Q}^{*}}\left\|\mathrm{~b}_{2}(z)-\left(\mathrm{b}_{2}\right)_{\mathrm{Q}^{*}}\right\| \mathrm{T}\left(\mathrm{f}_{1}^{\infty}, \mathrm{f}_{2}^{\infty}\right)(z)\right| \mathrm{d} z
$$




$$
\begin{aligned}
& +\mathrm{C} \frac{1}{|\mathrm{Q}|} \int_{\mathrm{Q}}\left|\mathrm{b}_{1}(z)-\left(\mathrm{b}_{1}\right)_{\mathrm{Q}^{*}}\right| \mathrm{T}\left(\mathrm{f}_{1}^{\infty},\left(\mathrm{b}_{2}-\left(\mathrm{b}_{2}\right)_{\mathrm{Q}^{*}}\right) \mathrm{f}_{2}^{\infty}\right)(z) \mid \mathrm{d} z \\
& +\mathrm{C} \frac{1}{|\mathrm{Q}|} \int_{\mathrm{Q}}\left|\mathrm{b}_{2}(z)-\left(\mathrm{b}_{2}\right)_{\mathrm{Q}^{*}}\right|\left|\mathrm{T}\left(\left(\mathrm{b}_{1}-\left(\mathrm{b}_{1}\right)_{\mathrm{Q}^{*}}\right) \mathrm{f}_{1}^{\infty}, \mathrm{f}_{2}^{\infty}\right)(z)\right| \mathrm{d} z \\
& +\mathrm{C} \frac{1}{|\mathrm{Q}|} \int_{\mathrm{Q}}\left|\mathrm{T}\left(\left(\mathrm{b}_{1}-\left(\mathrm{b}_{1}\right)_{*}\right) \mathrm{f}_{1}^{\infty},\left(\mathrm{b}_{2}-\left(\mathrm{b}_{2}\right)_{*}\right) \mathrm{f}_{2}^{\infty}\right)(z)-\mathrm{C}_{\mathrm{Q}}\right| \mathrm{d} z \\
& =\mathrm{B}_{41}+\mathrm{B}_{42}+\mathrm{B}_{43}+\mathrm{B}_{44} .
\end{aligned}
$$

Since $B_{41}, B_{42}, B_{43}$ can be handled along the same way to estimate $B_{11}$, we only need to compute the fourth term. Similar to estimate $A_{44}$ with $p_{1}=\frac{n}{\beta_{1}}, p_{2}=\frac{n}{\beta_{2}}$, we have

$$
\begin{aligned}
& \left.\mathrm{B}_{44} \leqslant \mathrm{C} \frac{1}{|\mathrm{Q}|} \int_{\mathrm{Q}} \sum_{\mathrm{j}, \mathrm{k} \geqslant 1}\left(\int_{S_{j}\left(\mathrm{Q}^{*}\right) \times \mathrm{S}_{\mathrm{k}}\left(\mathrm{Q}^{*}\right)} \int_{0}^{\infty}\left|\mathrm{K}_{\mathrm{t}}\left(z, \mathrm{y}_{1}, \mathrm{y}_{2}\right)-\mathrm{K}_{\mathrm{t}}\left(\mathrm{x}_{\mathrm{Q}}, \mathrm{y}_{1}, \mathrm{y}_{2}\right)\right|^{2} \frac{\mathrm{dt}}{\mathrm{t}}\right)^{\frac{\mathrm{p}_{0}}{2}} \mathrm{~d} \mathrm{y}_{1} \mathrm{~d} \mathrm{y}_{2}\right)^{1 / \mathrm{p}_{0}} \mathrm{~d} z \\
& \times\left(\left.\int_{2^{j} Q^{*}}\left(b_{1}\left(y_{1}\right)-\left(b_{1}\right)_{*}\right)\right|^{p_{0}}\left|f_{1}\left(y_{1}\right)\right|^{p_{0}} d y_{1}\right)^{\frac{1}{p_{0}}}\left(\left.\int_{2^{k} Q^{*}}\left(b_{2}\left(y_{2}\right)-\left(b_{2}\right)_{*}\right)\right|^{p_{0}}\left|f_{2}\left(y_{2}\right)\right|^{p_{0}} d y_{2}\right)^{\frac{1}{p_{0}}} \\
& \leqslant\left.\sum_{j_{0} \geqslant 1} \mathrm{C} \frac{1}{|\mathrm{Q}|} \int_{\mathrm{Q}} \frac{\left|x_{\mathrm{Q}}-z\right|^{2\left(\delta-\frac{n}{\mathrm{p}_{0}}\right)}}{\left|\mathrm{Q}^{*}\right|^{\frac{2 \delta}{n}}} 2^{-2 \delta j_{0}} \mathrm{~d} z\left|2^{j_{0}} \mathrm{Q}^{*}\right|^{\frac{\beta_{1}}{n}}\left\|\mathrm{~b}_{1}\right\|_{{\dot{\beta_{\beta}}}_{\beta_{1}}} 2^{\mathrm{j}_{0}} \mathrm{Q}^{*}\right|^{\frac{1}{p_{0}}}-\frac{\beta_{1}}{n}\left\|\mathrm{f}_{1}\right\|_{\mathrm{L}^{\frac{n}{\beta_{1}}}} \\
& \times\left|2^{j_{0}} Q^{*}\right|^{\frac{\beta_{2}}{n}}\left\|b_{2}\right\|_{i_{\beta_{2}}}\left|2^{j_{0}} Q^{*}\right|^{\frac{1}{p_{0}}-\frac{\beta_{2}}{n}}\left\|f_{2}\right\|_{L^{\frac{n}{\beta_{2}}}} \\
& \leqslant C \prod_{j=1}^{2}\left\|b_{j}\right\|_{\dot{\Lambda}_{\beta_{j}}}\left\|f_{1}\right\|_{L^{\frac{n}{\beta_{1}}}}\left\|f_{2}\right\|_{L^{\frac{n}{\beta_{2}}}} \sum_{j_{0} \geqslant 1}|Q|^{-\frac{2}{p_{0}}} 2^{-2 j_{0}\left(\delta-\frac{n}{p_{0}}\right)}\left|Q^{*}\right|^{\frac{2}{p_{0}}} \\
& \leqslant C \prod_{j=1}^{2}\left\|b_{j}\right\|_{\lambda_{\beta_{j}}}\left\|f_{1}\right\|_{L^{\frac{n}{\beta_{1}}}}\left\|f_{2}\right\|_{L^{\frac{n}{\beta_{2}}}}
\end{aligned}
$$

where $\delta$ must satisfy $\delta>\frac{n}{p_{0}}$.

From the estimates of $\mathrm{B}_{1}, \mathrm{~B}_{2}, \mathrm{~B}_{3}$ and $\mathrm{B}_{4}$, one follows that

$$
\left\|T_{\Pi b}\left(f_{1}, f_{2}\right)\right\|_{\text {BMO }} \leqslant C\left\|b_{1}\right\|_{{\dot{\lambda_{1}}}_{\beta_{1}}}\left\|b_{2}\right\|_{\dot{\lambda}_{\beta_{2}}}\left\|f_{1}\right\|_{L^{\frac{n}{\beta_{1}}}}\left\|f_{2}\right\|_{L^{\frac{n}{\beta_{2}}}} .
$$

\section{Boundedness of iterated commutators on Triebel-Lizorkin spaces}

Along the same line to prove Theorem 1.4, we also consider the case $m=2$, since the argument for the case $m>2$ is similar.

Lemma 6.1. Let $\mathrm{T}_{\Pi \mathrm{b}}$ be the iterated commutator of multilinear square function defined in (1.1) with kernel $\mathrm{K}_{\mathrm{t}}$ satisfying the conditions $\left(\mathrm{H}_{1}\right),\left(\mathrm{H}_{2}\right),\left(\mathrm{H}_{3}\right)$ and $\left(\mathrm{H}_{4}\right)$. Assume that $\mathrm{b}_{j} \in \dot{\Lambda}_{\beta_{j}}$ for $j=1,2$ satisfying $0<\beta_{j}, \beta<$ $1, \beta=\sum_{j=1}^{2} \beta_{j}$. We further assume that $\delta>\frac{\beta}{2}-\frac{n}{p_{0}}$ and some $s_{3}>1$. Then

$$
\begin{aligned}
& \frac{1}{|Q|^{1+\frac{\beta}{n}}} \int_{Q}\left|T_{\Pi b}\left(f_{1}, f_{2}\right)(z)-\left(T_{\Pi b}\left(f_{1}, f_{2}\right)\right)_{Q}\right| d z \\
& \quad \leqslant C \prod_{j=1}^{2}\left\|b_{j}\right\|_{\lambda_{\beta_{j}}}\left[M_{s_{3}}\left(T\left(f_{1}, f_{2}\right)\right)(x)+M_{p_{0}}\left(f_{1}\right)(x) M_{p_{0}}\left(f_{2}\right)(x)\right] .
\end{aligned}
$$

Proof. Fix $Q$ to be any cube with its center $x_{Q}$ and side length $\ell$. Let $f_{i}^{0}, f_{i}^{\infty}, i=1,2$ and $Q^{*}$ be as in the 
proof of Theorem 1.4. Then we write

$$
\begin{aligned}
\frac{1}{|\mathrm{Q}|^{1+\frac{\beta}{n}}} \int_{\mathrm{Q}} \mid \mathrm{T}_{\Pi \mathrm{b}}\left(\mathrm{f}_{1}, \mathrm{f}_{2}\right)(z)- & \left(\mathrm{T}_{\Pi \mathrm{b}}\left(\mathrm{f}_{1}, \mathrm{f}_{2}\right)\right)_{\mathrm{Q}} \mid \mathrm{d} z \\
\leqslant & C \frac{1}{|\mathrm{Q}|^{1+\frac{\beta}{n}}} \int_{\mathrm{Q}}\left|\mathrm{b}_{1}(z)-\left(\mathrm{b}_{1}\right)_{\mathrm{Q}^{*}}\left\|\mathrm{~b}_{2}(z)-\left(\mathrm{b}_{2}\right)_{\mathrm{Q}^{*}}\right\| \mathrm{T}\left(\mathrm{f}_{1}, \mathrm{f}_{2}\right)(z)\right| \mathrm{d} z \\
+ & \mathrm{C} \frac{1}{|\mathrm{Q}|^{1+\frac{\beta}{n}}} \int_{\mathrm{Q}}\left|\mathrm{b}_{1}(z)-\left(\mathrm{b}_{1}\right)_{\mathrm{Q}^{*}}\right|\left|\mathrm{T}\left(\mathrm{f}_{1},\left(\mathrm{~b}_{2}(z)-\left(\mathrm{b}_{2}\right)_{\mathrm{Q}^{*}}\right) \mathrm{f}_{2}\right)(z)\right| \mathrm{d} z \\
+ & \mathrm{C} \frac{1}{|\mathrm{Q}|^{1+\frac{\beta}{n}}} \int_{\mathrm{Q}}\left|\mathrm{b}_{2}(z)-\left(\mathrm{b}_{2}\right)_{\mathrm{Q}^{*}}\right|\left|\mathrm{T}\left(\left(\mathrm{b}_{1}(z)-\left(\mathrm{b}_{1}\right)_{\mathrm{Q}^{*}}\right) \mathrm{f}_{1}, \mathrm{f}_{2}\right)(z)\right| \mathrm{d} z \\
+ & \frac{1}{|\mathrm{Q}|^{1+\frac{\beta}{n}}} \int_{\mathrm{Q}} \mid \mathrm{T}\left(\left(\mathrm{b}_{1}-\left(\mathrm{b}_{1}\right)_{\mathrm{Q}^{*}}\right) \mathrm{f}_{1},\left(\mathrm{~b}_{2}-\left(\mathrm{b}_{2}\right)_{\mathrm{Q}^{*}}\right) \mathrm{f}_{2}\right)(z) \\
& -\mathrm{T}\left(\left(\mathrm{b}_{1}-\left(\mathrm{b}_{1}\right)_{\mathrm{Q}^{*}}\right) \mathrm{f}_{1}^{\infty},\left(\mathrm{b}_{2}-\left(\mathrm{b}_{2}\right)_{\mathrm{Q}^{*}}\right) \mathrm{f}_{2}^{\infty}\right)\left(\mathrm{x}_{\mathrm{Q}}\right) \mid \mathrm{d} z \\
= & \mathrm{D}_{1}+\mathrm{D}_{2}+\mathrm{D}_{3}+\mathrm{D}_{4} .
\end{aligned}
$$

Thanks to Hölder's inequality and Lemma 2.2, we have

$$
\begin{aligned}
\mathrm{D}_{1} \leqslant & C\left(\frac{1}{|\mathrm{Q}|^{1+\frac{s_{1} \beta_{1}}{n}}} \int_{\mathrm{Q}}\left|\mathrm{b}_{1}(z)-\left(\mathrm{b}_{1}\right)_{\mathrm{Q}^{*}}\right|^{s_{1}} \mathrm{~d} z\right)^{\frac{1}{s_{1}}}\left(\frac{1}{|\mathrm{Q}|^{1+\frac{s_{2} \beta_{2}}{n}}} \int_{\mathrm{Q}}\left|\mathrm{b}_{2}(z)-\left(\mathrm{b}_{2}\right)_{\mathrm{Q}^{*}}\right|^{s_{2}} \mathrm{~d} z\right)^{\frac{1}{s_{2}}} \\
& \times\left(\frac{1}{|\mathrm{Q}|} \int_{\mathrm{Q}}\left|\mathrm{T}\left(\mathrm{f}_{1}, \mathrm{f}_{2}\right)(z)\right|^{s_{3}} \mathrm{~d} z\right)^{\frac{1}{s_{3}}} \\
\leqslant & C\left\|\mathrm{~b}_{1}\right\|_{\dot{\Lambda}_{\beta_{1}}}\left\|\mathrm{~b}_{2}\right\|_{{\dot{\Lambda_{\beta}}}_{\beta_{2}}} \mathrm{M}_{\mathrm{s}_{3}}\left(\mathrm{~T}\left(\mathrm{f}_{1}, \mathrm{f}_{2}\right)\right)(\mathrm{x})
\end{aligned}
$$

with $\frac{1}{s_{1}}+\frac{1}{s_{2}}+\frac{1}{s_{3}}=1$.

We split $\mathrm{D}_{3}$ into the following term.

$$
\begin{aligned}
\mathrm{D}_{3} \leqslant & C \frac{1}{|\mathrm{Q}|^{1+\frac{\beta}{n}}} \int_{\mathrm{Q}}\left|\mathrm{b}_{2}(z)-\left(\mathrm{b}_{2}\right)_{\mathrm{Q}^{*}} \| \mathrm{T}\left(\left(\mathrm{b}_{1}-\left(\mathrm{b}_{1}\right)_{\mathrm{Q}^{*}}\right) \mathrm{f}_{1}^{0}, \mathrm{f}_{2}^{0}\right)(z)\right| \mathrm{d} z \\
& +\frac{1}{|\mathrm{Q}|^{1+\frac{\beta}{n}}} \int_{\mathrm{Q}}\left|\mathrm{b}_{2}(z)-\left(\mathrm{b}_{2}\right)_{\mathrm{Q}^{*}} \| \mathrm{T}\left(\left(\mathrm{b}_{1}-\left(\mathrm{b}_{1}\right)_{\mathrm{Q}^{*}}\right) \mathrm{f}_{1}^{0}, \mathrm{f}_{2}^{\infty}\right)(z)\right| \mathrm{d} z \\
& +\frac{1}{|\mathrm{Q}|^{1+\frac{\beta}{n}}} \int_{\mathrm{Q}}\left|\mathrm{b}_{2}(z)-\left(\mathrm{b}_{2}\right)_{\mathrm{Q}^{*}} \| \mathrm{T}\left(\left(\mathrm{b}_{1}-\left(\mathrm{b}_{1}\right)_{\mathrm{Q}^{*}}\right) \mathrm{f}_{1}^{\infty}, \mathrm{f}_{2}^{0}\right)(z)\right| \mathrm{d} z \\
& +\frac{1}{|\mathrm{Q}|^{1+\frac{\beta}{n}}} \int_{\mathrm{Q}}\left|\mathrm{b}_{2}(z)-\left(\mathrm{b}_{2}\right)_{\mathrm{Q}^{*}} \| \mathrm{T}\left(\left(\mathrm{b}_{1}-\left(\mathrm{b}_{1}\right)_{\mathrm{Q}^{*}}\right) \mathrm{f}_{1}^{\infty}, \mathrm{f}_{2}^{\infty}\right)(z)\right| \mathrm{d} z \\
= & \mathrm{D}_{31}+\mathrm{D}_{32}+\mathrm{D}_{33}+\mathrm{D}_{34} .
\end{aligned}
$$

Similar to estimate $\mathrm{B}_{21}$, the condition $\left(\mathrm{H}_{3}\right),(2.1)$ and Hölder's inequality give that

$$
\begin{aligned}
& D_{33} \leqslant C\left\|b_{2}\right\|_{\dot{\Lambda}_{\beta_{1}}} \sum_{k=1}^{\infty} \frac{1}{|Q|^{1+\frac{\beta_{1}}{n}}} \int_{Q}\left(\int_{S_{k}\left(Q^{*}\right) \times S_{0}\left(Q^{*}\right)}\left(\int_{0}^{\infty}\left|K_{t}\left(z, y_{1}, y_{2}\right)\right|^{2} \frac{d t}{t}\right)^{\frac{p_{0}}{2}} d y_{1} d y_{2}\right)^{\frac{1}{p_{0}}} d z \\
& \times\left(\int_{2^{k} Q^{*}}\left|\left(b_{1}\left(y_{1}\right)-\left(b_{1}\right) Q^{*}\right)\right|^{p_{0}}\left|f_{1}\left(y_{1}\right)\right|^{p_{0}} d y_{1}\right)^{\frac{1}{p_{0}}}\left(\int_{Q^{*}}\left|f_{2}\left(y_{2}\right)\right|^{p_{0}} d y_{2}\right)^{\frac{1}{p_{0}}} \\
& \leqslant C\left\|b_{1}\right\|_{\dot{\Lambda}_{\beta_{1}}}\left\|b_{2}\right\|_{\dot{\Lambda}_{\beta_{2}}} \sum_{k=1}^{\infty} \frac{1}{\mid Q^{1+\frac{\beta_{1}}{n}}} \int_{Q} \frac{2^{-\frac{2 n k}{p_{0}}}}{\left|Q^{*}\right|^{\frac{2}{p_{0}}}} d z\left|2^{k} Q^{*}\right|^{\frac{\beta_{1}}{n}+\frac{1}{p_{0}}}
\end{aligned}
$$




$$
\begin{aligned}
& \times\left(\frac{1}{\left|2^{k} Q^{*}\right|} \int_{2^{k} Q^{*}}\left|f_{1}\left(y_{1}\right)\right|^{p_{0}} d y_{1}\right)^{\frac{1}{p_{0}}}\left(\int_{Q^{*}}\left|f_{2}\left(y_{2}\right)\right|^{p_{0}} d y_{2}\right)^{\frac{1}{p_{0}}} \\
\leqslant & C\left\|b_{1}\right\|_{\dot{\lambda}_{\beta_{1}}}\left\|b_{2}\right\|_{\dot{\lambda}_{\beta_{2}}} M_{p_{0}}\left(f_{1}\right)(x) M_{p_{0}}(f)(x) \sum_{k=1}^{\infty} 2^{-k\left(\frac{\mathfrak{n}}{p_{0}}-\beta_{1}\right)} \\
\leqslant & C\left\|b_{1}\right\|_{\dot{\lambda}_{\beta_{1}}}\left\|b_{2}\right\|_{{\dot{\lambda_{\beta}}}_{\beta_{2}}} M_{p_{0}}\left(f_{1}\right)(x) M_{p_{0}}(f)(x),
\end{aligned}
$$

where $\delta$ must satisfy $\beta_{1}<\frac{n}{p_{0}}$.

The same argument to $\mathrm{D}_{33}$, we deduce that

$$
D_{3 i} \leqslant C\left\|b_{1}\right\|_{{\dot{\beta_{\beta}}}_{1}}\left\|b_{2}\right\|_{{\dot{\lambda_{\beta}}}_{\beta_{2}}} M_{p_{0}}\left(f_{1}\right)(x) M_{p_{0}}(f)(x)
$$

for $i=1,2,4$, which imply

$$
D_{3} \leqslant C\left\|b_{1}\right\|_{i_{\beta_{1}}}\left\|b_{2}\right\|_{i_{\beta_{2}}} M_{p_{0}}\left(f_{1}\right)(x) M_{p_{0}}(f)(x)
$$

Similarly, one has

$$
D_{2} \leqslant C\left\|b_{1}\right\|_{i_{\beta_{1}}}\left\|b_{2}\right\|_{{\dot{\beta_{2}}}_{\beta_{2}}} M_{p_{0}}\left(f_{1}\right)(x) M_{p_{0}}(f)(x) .
$$

At last, we need to handle the fourth term.

$$
\begin{aligned}
\mathrm{D}_{4} \leqslant & C \frac{1}{|\mathrm{Q}|^{1+\frac{\beta}{n}}} \int_{\mathrm{Q}}\left|\mathrm{T}\left(\left(\mathrm{b}_{1}-\left(\mathrm{b}_{1}\right) \mathrm{Q}^{*}\right) \mathrm{f}_{1}^{0},\left(\mathrm{~b}_{2}-\left(\mathrm{b}_{2}\right) \mathrm{Q}^{*}\right) \mathrm{f}_{2}^{0}\right)(z)\right| \mathrm{d} z \\
& +\mathrm{C} \frac{1}{|\mathrm{Q}|^{1+\frac{\beta}{n}}} \int_{\mathrm{Q}}\left|\mathrm{T}\left(\left(\mathrm{b}_{1}-\left(\mathrm{b}_{1}\right)_{\mathrm{Q}^{*}}\right) \mathrm{f}_{1}^{0},\left(\mathrm{~b}_{2}-\left(\mathrm{b}_{2}\right)_{\mathrm{Q}^{*}}\right) \mathrm{f}_{2}^{\infty}\right)(z)\right| \mathrm{d} z \\
& +\mathrm{C} \frac{1}{|\mathrm{Q}|^{1+\frac{\beta}{n}}} \int_{\mathrm{Q}}\left|\mathrm{T}\left(\left(\mathrm{b}_{1}-\left(\mathrm{b}_{1}\right)_{\mathrm{Q}^{*}}\right) \mathrm{f}_{1}^{\infty},\left(\mathrm{b}_{2}-\left(\mathrm{b}_{2}\right)_{\mathrm{Q}^{*}}\right) \mathrm{f}_{2}^{0}\right)(z)\right| \mathrm{d} z \\
& +\mathrm{C} \frac{1}{|\mathrm{Q}|^{1+\frac{\beta}{n}}} \int_{\mathrm{Q}} \mid \mathrm{T}\left(\left(\mathrm{b}_{1}-\left(\mathrm{b}_{1}\right)_{\mathrm{Q}^{*}}\right) \mathrm{f}_{1}^{\infty},\left(\mathrm{b}_{2}-\left(\mathrm{b}_{2}\right)_{\mathrm{Q}^{*}}\right) \mathrm{f}_{2}^{\infty}\right)(z) \\
& -\mathrm{T}\left(\left(\mathrm{b}_{1}-\left(\mathrm{b}_{1}\right)_{\mathrm{Q}^{*}}\right) \mathrm{f}_{1}^{\infty},\left(\mathrm{b}_{2}-\left(\mathrm{b}_{2}\right)_{\mathrm{Q}^{*}}\right) \mathrm{f}_{2}^{\infty}\right)\left(\mathrm{x}_{\mathrm{Q}}\right) \mid \mathrm{d} z \\
= & \mathrm{D}_{41}+\mathrm{D}_{42}+\mathrm{D}_{43}+\mathrm{D}_{44} .
\end{aligned}
$$

Following the same line to estimate $A_{41}$, the condition $\left(\mathrm{H}_{4}\right)$ and Hölder's inequality read that

$$
\begin{aligned}
D_{41} \leqslant & C \frac{1}{\mid Q^{1+\frac{\beta}{n}}} \int_{Q}\left(\int_{S_{1}\left(Q^{*}\right) \times S_{1}\left(Q^{*}\right)}\left(\int_{0}^{\infty}\left|K_{t}\left(z, y_{1}, y_{2}\right)\right| \frac{d t}{t}\right)^{\frac{p_{0}}{2}} d y_{1} d y_{2}\right)^{\frac{1}{p_{0}}} d z \\
& \times \prod_{j=1}^{2}\left(\int_{Q^{*}} \mid\left(b_{j}-\left.\left(b_{j}\right)_{Q^{*}}\right|^{p_{0}}\left|f_{j}\left(y_{j}\right)\right|^{p_{0}} d y_{j}\right)^{\frac{1}{p_{0}}}\right. \\
\leqslant & C \prod_{j=1}^{2}\left\|b_{j}\right\|_{\lambda_{\beta_{j}}} \frac{1}{\mid Q^{1+\frac{\beta}{n}}} \int_{Q} \frac{1}{\left|Q^{*}\right|^{2 / p_{0}}} d z\left|Q^{*}\right|^{\frac{\beta}{n}} \prod_{j=1}^{2}\left(\int_{Q^{*}}\left|f_{j}\left(y_{j}\right)\right|^{p_{0}} d y_{j}\right)^{\frac{1}{p_{0}}} \\
\leqslant & C\left\|b_{1}\right\|_{\lambda_{\beta_{1}}}\left\|b_{2}\right\|_{\lambda_{\beta_{2}}} M_{p_{0}}\left(f_{1}\right)(x) M_{p_{0}}\left(f_{2}\right)(x),
\end{aligned}
$$

and

$$
D_{42} \leqslant C \frac{1}{|Q|^{1+\frac{\beta}{n}}} \int_{Q} \sum_{k=1}^{\infty}\left(\int_{S_{k}\left(Q^{*}\right) \times S_{0}\left(Q^{*}\right)}\left(\int_{0}^{\infty}\left|K_{t}\left(z, y_{1}, y_{2}\right)\right|^{2} \frac{d t}{t}\right)^{\frac{p_{0}^{\prime}}{2}} d y_{1} d y_{2}\right)^{1 / p_{0}^{\prime}} d z
$$




$$
\begin{aligned}
& \times\left(\int_{2^{k} Q^{*} \backslash 2^{k-1} Q^{*}}\left|\left(b_{1}\left(y_{1}\right)-\left(b_{1}\right)\right)_{Q^{*}}\right|^{p_{0}}\left|f_{1}\left(y_{1}\right)\right|^{p_{0}} d y_{1}\right)^{1 / p_{0}} \\
& \times\left(\int_{Q^{*}}\left|\left(b_{2}\left(y_{2}\right)-\left(b_{2}\right)\right)_{Q^{*}}\right|^{p}\left|f_{2}\left(y_{2}\right)\right|^{p_{0}} d y_{2}\right)^{1 / p_{0}} \\
\leqslant & \left.C\left\|b_{1}\right\|_{\dot{\lambda}_{\beta_{1}}}\left\|b_{2}\right\|_{\lambda_{\beta_{2}}}\left|Q^{-\frac{\beta}{n}} \sum_{k=1}^{\infty} \frac{2^{-2 k n / p_{0}}}{\left|Q^{*}\right|^{\frac{2}{p_{0}}}}\right| 2^{k} Q^{*}\right|^{\frac{\beta_{1}}{n}}\left|Q^{*}\right|^{\frac{\beta_{2}}{n}} \\
& \times\left(\int_{2^{k} Q^{*}}\left|f_{1}\left(y_{1}\right)\right|^{p_{0}} d y_{1}\right)^{1 / p_{0}}\left(\int_{Q^{*}}\left|f_{2}\left(y_{2}\right)\right|^{p_{0}} d y_{2}\right)^{1 / p_{0}} \\
\leqslant & C\left\|b_{1}\right\|_{\dot{\lambda}_{\beta_{1}}}\left\|b_{2}\right\|_{\lambda_{\beta_{2}}} M_{p_{0}}\left(f_{1}\right)(x) M_{p_{0}}\left(f_{2}\right)(x) \sum_{k=1}^{\infty} 2^{-k n\left(\frac{1}{p_{0}}-\frac{\beta_{1}}{n}\right)} \\
\leqslant & C\left\|b_{1}\right\|_{\dot{\lambda}_{\beta_{1}}}\left\|b_{2}\right\|_{\lambda_{\beta_{2}}} M_{p_{0}}\left(f_{1}\right)(x) M_{p_{0}}\left(f_{2}\right)(x) .
\end{aligned}
$$

Here it is based on the fact that $p_{0}<\frac{\eta}{\beta_{1}}$.

Following the line to estimate $\mathrm{D}_{42}$, we have

$$
\begin{aligned}
D_{43} & \leqslant C\left\|b_{1}\right\|_{{\dot{\beta_{1}}}_{1}}\left\|b_{2}\right\|_{{\dot{\lambda_{\beta}}}_{\beta_{2}}} M_{p_{0}}\left(f_{1}\right)(x) M_{p_{0}}\left(f_{2}\right)(x) \sum_{k=1}^{\infty} 2^{-k\left(\frac{n}{p_{0}}-\beta_{2}\right)} \\
& \leqslant C\left\|b_{1}\right\|_{{\dot{\lambda_{\beta}}}_{\beta_{1}}}\left\|b_{2}\right\|_{\dot{\Lambda}_{\beta_{2}}} M_{p_{0}}\left(f_{1}\right)(x) M_{p_{0}}\left(f_{2}\right)(x)
\end{aligned}
$$

which used the condition $p_{0}<\frac{n}{\beta_{2}}$.

Finally, the same argument as $A_{44}$ yields that

$$
\begin{aligned}
& D_{44} \leqslant C \frac{1}{|Q|^{1+\frac{\beta}{n}}} \int_{Q_{j, k \geqslant 1}}\left(\int_{S_{j}\left(Q^{*}\right) \times S_{k}\left(Q^{*}\right)}\left(\int_{0}^{\infty}\left|K_{t}\left(z, y_{1}, y_{2}\right)-K_{t}\left(x_{Q}, y_{1}, y_{2}\right)\right|^{2} \frac{d t}{t}\right)^{\frac{p_{0}^{\prime}}{2}} d y_{1} d y_{2}\right)^{\frac{1}{p_{0}^{\prime}}} d z \\
& \times\left(\int_{2^{j} Q^{*}}\left|b_{1}\left(y_{1}\right)-\left(b_{1}\right)_{Q^{*}}\right|^{p_{0}}\left|f_{1}\left(y_{1}\right)\right|^{p_{0}} d y_{1}\right)^{1 / p_{0}}\left(\int_{2^{k} Q^{*}}\left|b_{2}\left(y_{2}\right)-\left(b_{2}\right)_{Q^{*}}\right|^{p_{0}}\left|f_{2}\left(y_{2}\right)\right|^{p_{0}} d y_{2}\right)^{1 / p_{0}} \\
& \left.\leqslant C\left\|b_{1}\right\|_{\dot{\Lambda}_{\beta_{1}}}\left\|b_{2}\right\|_{\dot{\Lambda}_{\beta_{2}}} \frac{1}{\mid Q^{1+\frac{\beta}{n}}} \sum_{j_{0} \geqslant 1} \int_{Q} \frac{\left|z-x_{Q}\right|^{2\left(\delta-n / p_{0}\right)}}{\left|Q^{*}\right|^{2 \delta / n}}\right) 2^{-2 \delta j_{0}} d z \\
& \times\left|2^{j_{0}} Q^{*}\right|^{\frac{\beta_{1}}{n}}\left(\int_{2^{j_{0}} Q^{*}}\left|f_{1}\left(y_{1}\right)\right|^{p_{0}} d y_{1}\right)^{\frac{1}{p_{0}}}\left|2^{j_{0}} Q^{*}\right|^{\frac{\beta_{2}}{n}}\left(\int_{2^{j} Q^{*}}\left|f_{2}\left(y_{2}\right)\right|^{p_{0}} d y_{2}\right)^{\frac{1}{p_{0}}} \\
& \leqslant C\left\|b_{1}\right\|_{\lambda_{\beta_{1}}}\left\|b_{2}\right\|_{\lambda_{\beta_{2}}} \sum_{j_{0} \geqslant 1} 2^{-j_{0}(2 \delta-\beta)}\left|Q^{*}\right|^{-\frac{2}{p_{0}}}\left(\int_{2^{j_{0}} Q^{*}}\left|f_{1}\left(y_{1}\right)\right|^{p_{0}} d y_{1}\right)^{1 / p_{0}}\left(\int_{2^{j_{0}} Q^{*}}\left|f_{2}\left(y_{2}\right)\right|^{p_{0}} d y_{2}\right)^{1 / p_{0}} \\
& \leqslant C\left\|b_{1}\right\|_{\dot{\Lambda}_{\beta_{1}}}\left\|b_{2}\right\|_{{\dot{\beta_{\beta}}}_{\beta_{2}}} M_{p_{0}}\left(f_{1}\right)(x) M_{p_{0}}\left(f_{2}\right)(x) \sum_{j_{0}>0} 2^{-j_{0}\left(2 \delta-\beta+\frac{2 n}{p_{0}}\right)} \\
& \leqslant C \prod_{j=1}^{2}\left\|b_{j}\right\|_{\dot{\Lambda}_{\beta_{j}}} M_{p_{0}}\left(f_{1}\right)(x) M_{p_{0}}\left(f_{2}\right)(x) .
\end{aligned}
$$

Here we used the condition $\delta>\frac{\beta}{2}-\frac{n}{p_{0}}$.

Combining the estimates of $\mathrm{D}_{41}-\mathrm{D}_{44}$, it follows that

$$
D_{4} \leqslant C \prod_{j=1}^{2}\left\|b_{j}\right\|_{{\dot{\beta_{\beta}}}_{j}} M_{p_{0}}\left(f_{1}\right)(x) M_{p_{0}}\left(f_{2}\right)(x),
$$


which together with the estimates of $D_{1}, D_{2}$ and $D_{3}$ gives that

$$
\begin{aligned}
& \frac{1}{|Q|^{1+\frac{\beta}{n}}} \int_{Q}\left|T_{\Pi b}\left(f_{1}, f_{2}\right)(z)-\left(T_{\Pi b}\left(f_{1}, f_{2}\right)\right)_{Q}\right| d z \\
& \quad \leqslant C \prod_{j=1}^{2}\left\|b_{j}\right\|_{\dot{\lambda}_{\beta_{j}}}\left[M_{s_{3}}\left(T\left(f_{1}, f_{2}\right)\right)(x)+M_{p_{0}}\left(f_{1}\right)(x) M_{p_{0}}\left(f_{2}\right)(x)\right] .
\end{aligned}
$$

Now we are in position to prove Theorem 1.6.

Proof of Theorem 1.6. Taking $\mathrm{L}^{\mathrm{p}}$-norm on both sides of (6.1) and apply the $\mathrm{L}^{\mathrm{p}}$-boundedness of the maximal operator $M_{s_{3}}, M_{p_{0}}$ and $\left(L^{p_{1}} \times L^{p_{2}}, L^{p}\right)$-boundedness of $T$, we have

$$
\begin{aligned}
\left\|T_{\Pi \mathbf{b}}\left(f_{1}, f_{2}\right)\right\|_{\dot{F}_{p}^{\beta, \infty}} & \approx\left\|\frac{1}{|Q|^{1+\frac{\beta}{n}}} \int_{Q}\left|T_{\Pi b}\left(f_{1}, f_{2}\right)(z)-\left(T_{\Pi b}\left(f_{1}, f_{2}\right)\right)_{Q}\right| d z\right\|_{L^{p}} \\
& \leqslant C \prod_{j=1}^{2}\left\|b_{j}\right\|_{\lambda_{\beta_{j}}}\left(\left\|M_{s_{3}}\left(T\left(f_{1}, f_{2}\right)\right)\right\|_{L^{p}}+\left\|M_{p_{0}}\left(f_{1}\right)(x) M_{p_{0}}\left(f_{2}\right)(x)\right\|_{L^{p}}\right) \\
& \leqslant C \prod_{j=1}^{2}\left\|b_{j}\right\|_{\lambda_{\beta_{j}}}\left(\left\|T\left(f_{1}, f_{2}\right)\right\|_{L^{p}}+\left\|M_{p_{0}}\left(f_{1}\right)\right\|_{L^{p_{1}}}\left\|M_{\mathfrak{p}_{0}}\left(f_{2}\right)\right\|_{L^{p_{2}}}\right) \\
& \leqslant C \prod_{j=1}^{2}\left\|b_{j}\right\|_{\lambda_{\beta_{j}}}\left\|f_{1}\right\|_{L^{p_{1}}}\left\|f_{2}\right\|_{L^{p_{2}}},
\end{aligned}
$$

which completes the proof of Theorem 1.6.

\section{Acknowledgment}

D. Chen is partially supported by NSF of China under Grant 11261023 and 11461033,11400129 , the Natural Science Foundation of Jiangxi Provience (20171BAB201003) and the foundation of CSC(201609470010) and CSC(201608360133).

\section{References}

[1] R. R. Coifman, Y. Meyer, On commutators of singular integrals and bilinear singular integrals, Trans. Amer. Math. Soc., 212 (1975), 315-331. 1

[2] R. R. Coifman, Y. Meyer, Au-delá des opérateurs pseudo-différentiels, Societe mathematique de France, Paris, (1978).

[3] R. R. Coifman, Y. Meyer, Commutateurs dintégrales singuliéres et opérateurs multilineaires, Ann. Inst. Fourier, 28 (1978), 177-202. 1

[4] E. B. Fabes, D. S. Jerison, C. E. Kenig, Multilinear Littlewood-Paley estimates with applications to partial differential equations, Proc. Natl. Acad. Sci., 79 (1982), 5746-5750. 1

[5] E. B. Fabes, D. S. Jerison, C. E. Kenig, Necessary and sufficient conditions for absolute continuity of elliptic harmonic measure, Ann. of Math., 119 (1984), 121-141.

[6] E. B. Fabes, D. S. Jerison, C. E. Kenig, Multilinear square functions and partial differential equations, Amer. J. Math., 107 (1985), 1325-1368. 1

[7] C. Fefferman, E. Stein, Some maximal inequalities, Amer. J. Math., 93 (1971), 107-115. 2

[8] L. Grafakos, On multilinear fractional integrals, Studia Math., 102 (1992), 49-56. 1, 2

[9] L. Grafakos, R. H. Torres, Maximal operator and weighted norm inequalities for multilinear singular integrals, Indiana Univ. Math. J., 51 (2002), 1261-1276. 1

[10] L. Grafakos, R. Torres, Multilinear Calderon-Zygmund theory, Adv. Math., 165 (2002), 124-164. 1

[11] C. E. Kenig, E. M. Stein, Multilinear estimates and fractional integration, Math. Res. Lett., 6 (1999), 1-15. 1

[12] A. K. Lerner, S. Ombrosi, C. Pérez, R. H. Torres, R. Trujillo-Gonzszlez, New maximal functions and multiple weight for the multilinear Caldeón-Zygmund theory, Advances in Mathematics, 220 (2009), 1222-1264. 3 
[13] W. Li, Q.-G. Xue, K. Yabuta, Weighted version of Carleson measure and multilinear Fourier multiplier, Forum Math., 27 (2015), 787-805. 1

[14] M. Paluszynski, Characterization of the Besov spaces via the commutator operator of Coifman Rochberg and Weiss, Indiana Univ Math J., 44 (1995), 1-17. 2

[15] S. Shi, Q. Xue, K. Yabuta, On the boundedness of multilinear Littlewood-Paley g $\mathrm{\lambda}_{\lambda}^{*}$ function, J. Math. Pures Appl., 101 (2014), 394-413. 1, 1

[16] L. Tang, Endpoint estimates for multilinear fractional integrals, J. Aust. Math. Soc., 84 (2008), 419-429. 1, 1.7

[17] Q. Xue, J. Yan, On multilinear square function and its applications to multilinear Littlewood-Paley operators with nonconvolution type kernels, J. Math. Anal. Appl., 422 (2015), 1342-1362. 1

[18] K. Yabuta, A multilinearization of Littlewood-Paley's g-function and Carleson measures, Tohoku Math. J., 34 (1982), 251-275. 1 